\title{
8-2018
}

\section{Finite-time Anti-synchronization of Memristive Stochastic BAM Neural Networks with Probabilistic Time-varying Delays}

\author{
Manman Yuan \\ University of Science and Technology Beijing \\ Weiping Wang \\ ersity of Science and Technology Beijing, weipingwangjt@ustb.edu.cn \\ Xiong Luo \\ ersity of Science and Technology Beijing \\ Linlin Liu \\ Beijing University of Techology \\ Wenbing Zhao \\ Cleveland State University, w.zhao1@csuohio.edu
}

Follow this and additional works at: https://engagedscholarship.csuohio.edu/enece_facpub

Part of the Electrical and Computer Engineering Commons

How does access to this work benefit you? Let us know!

\section{Repository Citation}

Yuan, Manman; Wang, Weiping; Luo, Xiong; Liu, Linlin; and Zhao, Wenbing, "Finite-time Antisynchronization of Memristive Stochastic BAM Neural Networks with Probabilistic Time-varying Delays" (2018). Electrical Engineering \& Computer Science Faculty Publications. 451.

https://engagedscholarship.csuohio.edu/enece_facpub/451

This Article is brought to you for free and open access by the Electrical Engineering \& Computer Science Department at EngagedScholarship@CSU. It has been accepted for inclusion in Electrical Engineering \& Computer Science Faculty Publications by an authorized administrator of EngagedScholarship@CSU. For more information, please contact library.es@csuohio.edu. 


\title{
Finite-time anti-synchronization of memristive stochastic BAM neural networks with probabilistic time-varying delays
}

\author{
Manman Yuan ${ }^{a, b}$, Weiping Wanga,b,c,*, Xiong Luo ${ }^{a, b, *}$, Linlin Liu ${ }^{d}$, Wenbing Zhao ${ }^{e}$ \\ a School of Computer and Communication Engineering, University of Science and Technology Beijing, Beijing 100083, China \\ ${ }^{\mathrm{b}}$ Beijing Key Laboratory of Knowledge Engineering for Materials Science, Beijing 100083, China \\ ${ }^{c}$ Institute of Physics, Humboldt-University, Berlin 10099, Germany \\ dInstitute of Microstructure and Properties of Advanced Materials, Beijing University of Technology, Beijing 100124, China \\ e Department of Electrical Engineering and Computer Science, Cleveland State University, Cleveland, OH 44115, USA
}

\author{
A R T I C L E I N F O \\ Article history: \\ Received 4 October 2017 \\ Revised 25 May 2018 \\ Accepted 11 June 2018 \\ Available online 23 June 2018 \\ Keywords: \\ Memristor \\ Stochastic BAM neural networks \\ Finite-time anti-synchronization \\ Probabilistic time-varying delays \\ Leakage delays
}

\begin{abstract}
A B S T R A C T
This paper investigates the drive-response finite-time anti-synchronization for memristive bidirectional associative memory neural networks (MBAMNNs). Firstly, a class of MBAMNNs with mixed probabilistic time-varying delays and stochastic perturbations is first formulated and analyzed in this paper. Secondly, an nonlinear control law is constructed and utilized to guarantee drive-response finite-time antisynchronization of the neural networks. Thirdly, by employing some inequality technique and constructing an appropriate Lyapunov function, some anti-synchronization criteria are derived. Finally, a number simulation is provided to demonstrate the effectiveness of the proposed mechanism.
\end{abstract}

\section{Introduction}

In the past decades, a large amount of attention has been devoted to the bidirectional associative memory neural networks (BAMNNs) owing to their potential applications from signal processing, pattern recognition, associative memory, and so on [1-5]. Nowadays, with the increasing amount of data [6,7] and complex neural networks (NNs) [8,9], scientists conceive that we can get a new type of BAMNNs named MBAMNNs, which the self-feedback connection weights are implemented by memristors [10-16] rather than resistances. From the system theoretic point of view, MBAMNNs can be treated as a class of state-depend nonlinear system [17], and it is a challenging topic. With the research and development of memristor, plenty of researchers from various fields show skyscraping enthusiasm to this kind of memristive neural networks (MNNs) [18-22]. The MBAMNNs have remarkable properties such as satisfactory convergence rate, computer implementation, a large number of equilibrium points, and so on [23]. Therefore, this type of model can better emulate the human brain than the traditional neural networks.
Synchronization, which means the dynamical signals of chaotic coupled system achieve an identical behavior with time moving. In reality, it is significant to consider the synchronization of its different potential applications including biological systems, intelligent control, secure communication, and image protection. As a typical collective behavior, the stability and synchronization of MNNs have been widely discussed, including lag synchronization [24], exponential synchronization, anti-synchronization [25-28], finite time synchronization [29,30], and so on. Recently, chaotic synchronization of MBAMNNs has gained much attention due to its successful applications in various areas [31-34].

However, in addition to this result, there are few results on the anti-synchronization for MBAMNNs. Additionally, among these synchronization works of MBAMNNs, most are asymptotic, implying the stability or synchronization of chaotic systems can be accomplished only when time towards to infinity. But from the point of practical, owing to the life span of human and machine [35], it is more pressing to achieve synchronization within the finite time, that is, finite time synchronization [37-40]. Besides, finite-time synchronization can illustrates the faster synchronous rate after a finite time-interval named setting time. Therefore, it is more practical and valuable to investigate the finite-time antisynchronization control of the MBAMNNs.

In the process of studying MBAMNNs, it is detected that the delays frequently appears owing to the limited transfer speed and the 
information processing. Actually, in the electronic implementation of analog NNs, time delay frequently occurring in the response and communication of neurons. And the time delay can lead to a series of questions, to a certain extent, affected the instability and oscillation to the NNs [41-44]. In the view of this, it is rewarding and reasonable to study the delayed NNs [45-49]. Nevertheless, time delays often occurring in a random way in the view of probabilistic reasons. This often occurs in real systems where the probability to taking vary large values of delay is very small [50].

Under these situations, probabilistic measurement delays would be regarded as a Bernoulli distributed white sequence [51] to depict more property of the real systems. Furthermore, it should not be neglected that in a real nervous system, there is a typical time delay named leakage delay which has tendency to impact on the dynamical behavior of NNs [52-54]. From the above discussions, it is significant and necessary to investigate some reasonable and practical systems for MBAMNNs. The actual communication between NNs is inevitably disturbed by a stochastic perturbation. The stochastic perturbation mainly comes from various uncertainties which probably results in package losses or influences the signal transmission. Hence, it is important to discuss the effect of probabilistic delays and stochastic perturbations. It should be mentioned that, the finite-time anti-synchronization results for stochastic MBAMNNs with probabilistic time-varying delays has not been studied yet, this motivates our present study.

Motivated by the aforementioned concerns, the aim of this paper is to investigate the finite-time anti-synchronization results for stochastic MBAMNNs with probabilistic time-varying delays. With the aid of the set-valued map, memristor mathematical model, differential inclusion, linear feedback controller, adaptive linear feedback controller, and the definition of anti- synchronization, two new sufficient criteria are derived to guarantee the finite-time anti-synchronization of MMAMNNs with mixed probabilistic timevarying delay. The main contributions of this paper can be summarized as follows.

1. We focus on the study of MBAMNNs models with stochastic perturbation and various time-varying delays, which including non-delay, discrete time-varying delays and a constant delay in the leakage term. Many other MBAMNNs models with delays are the special cases of our considered model.

2. We first attempt to address the finite-time anti-synchronization control problem for a class of proposed MBAMNNs models. By utilizing sign function and the definition of finite-time stability, a suitable nonlinear state feedback controller is designed. We consider and analysis the complex randomness of the timevarying delays rather than treat them as the same stability. Some main results are derived by utilizing the Lyapunov function, finite-time stability theorem, stochastic analysis theory and Wirtinger-type inequality.

3. Finally, we provided the numerical examples to illustrated the effectiveness and rationality of the proposed conclusions.

The rest of this paper is organized as follows. Some definitions, lemmas and assumptions about the proposed model are presented in Section 2. In Section 3 derives some sufficient conditions of finite time anti-synchronization based our considered MBAMNNs. Numerical simulations are demonstrated to verify the effectiveness of the obtained results in Section 4. Finally, the conclusion is given in Section 5.

Notations. For $r>0, \boldsymbol{C}\left([-r, 0], \mathbb{R}^{n}\right)$ denotes the Banach space of all continuous functions mapping $[-r .0]$ into $\mathbb{R}^{n}$ with $q$-norm or $\infty$-norm by the following forms, respectively. $\mathbb{R}^{n}$ denotes the $n$ dimensional Euclidean space. The superscript $T$ represents matrix or vector transposition. We define the norm of the vector as $\left\|x_{i}\right\|$ indicates the 2-norm of a vector $x_{i}$, i.e., $\left\|x_{i}\right\|=\left(\sum_{i=1}^{n} x_{i}^{2}\right)^{\frac{1}{2}}, \operatorname{co}[a, b]$ denotes the convex hull (closure) of $\{a, b\}$.

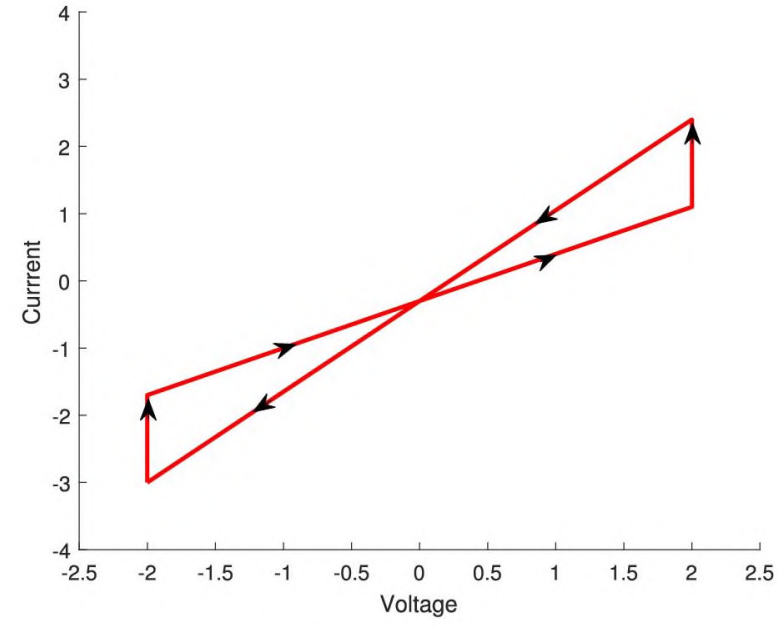

Fig. 1. Typical current-voltage characteristic of a memristor.

\section{Model description and preliminaries}

Based on the physical properties of memristor, a class of stochastic MBAMNNs with time-varying delays is described as follows

$$
\begin{aligned}
\dot{x}_{i}(t)= & -a_{i} x_{i}(t-\tau)+\sum_{j=1}^{m} b_{j i}\left(x_{i}(t)\right) f_{j}\left(y_{j}(t)\right) \\
& +\sum_{j=1}^{m} c_{j i}\left(x_{i}(t-\tau(t))\right) f_{j}\left(y_{j}(t-\tau(t))\right), \\
\dot{y}_{j}(t)= & -d_{j} y_{j}(t-\sigma)+\sum_{i=1}^{n} q_{i j}\left(y_{j}(t)\right) g_{i}\left(x_{i}(t)\right) \\
& +\sum_{i=1}^{n} p_{i j}\left(y_{j}(t-\sigma(t))\right) g_{i}\left(x_{i}(t-\sigma(t))\right),
\end{aligned}
$$

where $x_{i}(t)$ and $y_{j}(t)$ denote the voltages of capacitor $C_{i}$ and $\tilde{C}_{j}$ at time $t$, for $t \geq 0$ and $i=1,2, \ldots, n$ and $j=1,2, \ldots, m$. $a_{i}>0$ and $d_{j}>0$ represent the self-feedback connection weight. $f_{j}\left(y_{j}(t)\right)$ and $g_{i}\left(x_{i}(t)\right)$ are the feedback functions. $\tau(t)$ and $\sigma(t)$ are the time-varying delays which satisfied $0 \leq \tau(t) \leq \tau_{2}$ and $0 \leq \sigma(t) \leq \sigma_{2} . \quad b_{j i}\left(x_{i}(t)\right), \quad c_{j i}\left(x_{i}(t-\tau(t))\right), \quad q_{i j}\left(y_{j}(t)\right)$ and $p_{i j}\left(y_{j}(t-\right.$ $\sigma(t)))$ represent memristor-based weights, and $b_{j i}\left(x_{i}(t)\right)=\frac{W_{(1) j i}}{\tilde{c}_{j}} \times$ $\operatorname{sgin}_{j i}, \quad c_{j i}\left(x_{i}(t-\tau(t))\right)=\frac{W_{(2) j i}}{\bar{C}_{j}} \times \operatorname{sgin}_{j i}, \quad q_{i j}\left(y_{j}(t)\right)=\frac{W_{(3) i j}}{c_{i}} \times \operatorname{sgin}_{i j}$. $p_{i j}\left(y_{j}(t-\sigma(t))\right)=\frac{W_{(4) i j}}{C_{i}} \times \operatorname{sgin}_{i j}$,

$\operatorname{sgin}_{i j}=\left\{\begin{array}{l}1, i \neq j, \\ -1, i=j,\end{array} \quad \operatorname{sgin}_{j i}=\left\{\begin{array}{r}1, j \neq i, \\ -1, j=i,\end{array}\right.\right.$

then $W_{(1) j i}, W_{(2) j i}, W_{(3) i j}$ and $W_{(4) i j}$ denote the memductance of memristors $R_{(1) j i}, R_{(2) j i}, R_{(3) i j}$ and $R_{(4) i j}$, respectively. In addition, $R_{(1) j i}$ presents the memristor between the feedback function $f_{j}\left(y_{j}(t)\right)$ and $y_{j}(t), R_{(2) j i}$ presents the memristor between the feedback function $f_{j}\left(y_{j}(t-\tau(t))\right)$ and $y_{j}(t-\tau(t)), R_{(3) i j}$ presents the memristor between the feedback function $g_{i}\left(x_{i}(t)\right)$ and $x_{i}(t), R_{4}$ iji presents the memristor between the feedback function $g_{i}\left(x_{i}(t-\right.$ $\sigma(t)))$ and $x_{i}(t-\sigma(t))$.

According to the feature of memristor and the current-voltage characteristic, Fig. 1 can illustrates the simplification current characteristic of a memristor. And we apply the state-dependent parameters of the system (1) are satisfy the following conditions 
[51]:

$b_{j i}\left(x_{i}(t)\right)=\left\{\begin{aligned} \hat{b}_{j i}, & \left|x_{i}(t)\right|<\varpi_{i}, \\ \text { unchanged, } & \left|x_{i}(t)\right|=\varpi_{i}, \\ \tilde{b}_{j i}, & \left|x_{i}(t)\right|>\varpi_{i},\end{aligned}\right.$

$q_{i j}\left(y_{j}(t)\right)=\left\{\begin{aligned} \hat{q}_{i j}, & \left|y_{j}(t)\right|<\vartheta_{j}, \\ \text { unchanged, } & \left|y_{j}(t)\right|=\vartheta_{j}, \\ \check{q}_{i j}, & \left|y_{j}(t)\right|>\vartheta_{j} .\end{aligned}\right.$

$c_{j i}\left(x_{i}(t-\tau(t))\right)=\left\{\begin{aligned} \hat{c}_{j i}, & \left|x_{i}(t-\tau(t))\right|<\varpi_{i}, \\ \text { unchanged, } & \left|x_{i}(t-\tau(t))\right|=\varpi_{i}, \\ \check{c}_{j i}, & \left|x_{i}(t-\tau(t))\right|>\varpi_{i},\end{aligned}\right.$

$p_{i j}\left(y_{j}(t-\sigma(t))\right)=\left\{\begin{aligned} \hat{p}_{i j}, & \left|y_{j}(t-\sigma(t))\right|<\vartheta_{j}, \\ \text { unchanged, } & \left|y_{j}(t-\sigma(t))\right|=\vartheta_{j}, \\ \tilde{p}_{i j}, & \left|y_{j}(t-\sigma(t))\right|>\vartheta_{j},\end{aligned}\right.$

where $\sigma, \vartheta$ are nonnegative constants denoting the memristive switching jumps, and unchanged means that the memristance keeps the current value. $\hat{b}_{j i}, \check{b}_{j i}, \hat{c}_{j i}, \check{c}_{j i}, \hat{q}_{i j}, \check{q}_{i j}, \hat{p}_{i j}, \check{p}_{i j}$, are known constants relating to memristances.

Assuming system (1) as the drive system then the response system is:

$$
\begin{aligned}
d \hat{x}_{i}(t)= & {\left[-a_{i} \hat{x}_{i}(t-\tau)+\sum_{j=1}^{m} b_{j i}\left(\hat{x}_{i}(t)\right) f_{j}\left(\hat{y}_{j}(t)\right)\right.} \\
& \left.+\sum_{j=1}^{m} c_{j i}\left(\hat{x}_{i}(t-\tau(t))\right) f_{j}\left(\hat{y}_{j}(t-\tau(t))\right)+u_{i}(t)\right] d t \\
& +\sum_{j=1}^{m} \beta_{j i}\left(t, e_{j}(t), e_{j}(t-\tau(t))\right) d \omega_{j}(t), \\
d \hat{y}_{j}(t)= & {\left[-d_{j} \hat{y}_{j}(t-\sigma)+\sum_{i=1}^{n} q_{i j}\left(\hat{y}_{j}(t)\right) g_{i}\left(\hat{x}_{i}(t)\right)\right.} \\
& \left.+\sum_{i=1}^{n} p_{i j}\left(\hat{y}_{j}(t-\sigma(t))\right) g_{i}\left(\hat{x}_{i}(t-\sigma(t))\right)+u_{j}(t)\right] d t \\
& +\sum_{i=1}^{n} \beta_{i j}\left(t, e_{i}(t), e_{i}(t-\sigma(t))\right) d \omega_{i}(t),
\end{aligned}
$$

where $u_{i}(t)$ and $u_{j}(t)$ are the appropriate control inputs that will be designed, $\omega_{i}(t)$ and $\omega_{j}(t)$ are the Brownian motion defined on a complete probability space $(\Omega, \mathcal{F}, \mathcal{P})$.

Remark 1. According to the discussions above, the inner connection matrixes $b_{j i}\left(x_{i}(t)\right), q_{i j}\left(y_{j}(t)\right), c_{j i}\left(x_{i}(t-\tau(t))\right), p_{i j}\left(y_{j}(t-\sigma(t))\right)$ of system (1) and $b_{j i}\left(\hat{x}_{i}(t)\right), q_{i j}\left(\hat{y}_{j}(t)\right), c_{j i}\left(\hat{x}_{i}(t-\tau(t))\right), p_{i j}\left(\hat{y}_{j}(t-\right.$ $\sigma(t))$ ) of system (2) are all with the change of the memristance. Therefore, the proposed MBAMNNs are considered as the timevarying systems which depend on the state switching. When the inner connection matrixes are all constants, systems (1) and (2) become a general class of BAMNNs.

In this paper, the time-varying delays $\tau(t)$ and $\sigma(t)$ in system (1) are bounded with $0 \leq \tau(t) \leq \tau_{2}$ and $0 \leq \sigma(t) \leq \sigma_{2}$. In practice, there exist constants $\tau_{1}$ and $\sigma_{1}$ with $0 \leq \tau_{1} \leq \tau_{2}$ and $0 \leq \sigma_{1} \leq \sigma_{2}$. Furthermore, the probability distribution of $\tau(t)$ and $\sigma(t)$ taking values in $\left[0, \tau_{1}\right],\left(\tau_{1}, \tau_{2}\right],\left[0, \sigma_{1}\right]$ and $\left(\sigma_{1}, \sigma_{2}\right]$ with certain probability, respectively.

The information of probability distribution of $\tau(t)$ and $\sigma(t)$ are defined as

$\mathbb{P}\left\{\tau(t) \in\left[0, \tau_{1}\right]\right\}=\delta_{0}, \quad \mathbb{P}\left\{\tau(t) \in\left(\tau_{1}, \tau_{2}\right]\right\}=1-\delta_{0}$, $\mathbb{P}\left\{\sigma(t) \in\left[0, \sigma_{1}\right]\right\}=\rho_{0}, \quad \mathbb{P}\left\{\sigma(t) \in\left(\sigma_{1}, \sigma_{2}\right]\right\}=1-\rho_{0}$, where $0 \leq \delta_{0} \leq 1$ and $0 \leq \rho_{0} \leq 1$. Then

$$
\begin{gathered}
\tau(t)= \begin{cases}\tau_{1}(t), & \tau(t) \in\left[0, \tau_{1}\right], \\
\tau_{2}(t), & \tau(t) \in\left(\tau_{1}, \tau_{2}\right],\end{cases} \\
\sigma(t)= \begin{cases}\sigma_{1}(t), & \sigma(t) \in\left[0, \sigma_{1}\right], \\
\sigma_{2}(t), & \sigma(t) \in\left(\sigma_{1}, \sigma_{2}\right],\end{cases}
\end{gathered}
$$

and $\delta(t)$ and $\sigma(t)$ are the Bernoulli distributed sequence with

$$
\begin{aligned}
& \mathbb{P}\{\delta(t)=1\}=\mathbb{P}\left\{\tau(t) \in\left[0, \tau_{1}\right]\right\}=\mathbb{E}\{\delta(t)\}=\delta_{0}, \\
& \mathbb{P}\{\delta(t)=0\}=\mathbb{P}\left\{\tau(t) \in\left(\tau_{1}, \tau_{2}\right]\right\}=\mathbb{E}\{1-\delta(t)\}=1-\delta_{0}, \\
& \mathbb{P}\{\rho(t)=1\}=\mathbb{P}\left\{\sigma(t) \in\left[0, \sigma_{1}\right]\right\}=\mathbb{E}\{\rho(t)\}=\rho_{0}, \\
& \mathbb{P}\{\rho(t)=0\}=\mathbb{P}\left\{\sigma(t) \in\left(\sigma_{1}, \sigma_{2}\right]\right\}=\mathbb{E}\{1-\rho(t)\}=1-\rho_{0} .
\end{aligned}
$$

Furthermore, four time-varying delays $\tau_{1}(t), \tau_{2}(t), \sigma_{1}(t)$ and $\sigma_{2}(t)$ are introduced, such that

$$
\begin{gathered}
\tau(t)= \begin{cases}\tau_{1}(t), & \tau(t) \in\left[0, \tau_{1}\right], \\
\tau_{2}(t), & \tau(t) \in\left(\tau_{1}, \tau_{2}\right],\end{cases} \\
\sigma(t)= \begin{cases}\sigma_{1}(t), & \sigma(t) \in\left[0, \sigma_{1}\right], \\
\sigma_{2}(t), & \sigma(t) \in\left(\sigma_{1}, \sigma_{2}\right] .\end{cases}
\end{gathered}
$$

By employing the new functions $\tau_{1}(t), \tau_{2}(t), \sigma_{1}(t), \sigma_{2}(t)$ and the stochastic variables $\delta(t), \rho(t)$. Systems (1) and (2) can be rewritten under the theories of set-valued maps and differential inclusions above,

$$
\begin{aligned}
\dot{x}_{i}(t) \in & -a_{i} x_{i}(t-\tau)+\sum_{j=1}^{m} c o\left[b_{j i}\left(x_{i}(t)\right)\right] f_{j}\left(y_{j}(t)\right) \\
& +\delta(t) \sum_{j=1}^{m} \operatorname{co}\left[c_{j i}\left(x_{i}(t-\tau(t))\right)\right] f_{j}\left(y_{j}\left(t-\tau_{1}(t)\right)\right) \\
& +(1-\delta(t)) \sum_{j=1}^{m} \operatorname{co}\left[c_{j i}\left(x_{i}(t-\tau(t))\right)\right] f_{j}\left(y_{j}\left(t-\tau_{2}(t)\right)\right) \\
\dot{y}_{j}(t) \in & -d_{j} y_{j}(t-\sigma)+\sum_{i=1}^{n} \operatorname{co}\left[q_{i j}\left(y_{j}(t)\right)\right] g_{i}\left(x_{i}(t)\right) \\
& +\rho(t) \sum_{i=1}^{n} \operatorname{co}\left[p_{i j}\left(y_{j}(t-\sigma(t))\right)\right] g_{i}\left(x_{i}\left(t-\sigma_{1}(t)\right)\right) \\
& +(1-\rho(t)) \sum_{i=1}^{n} \operatorname{co}\left[p_{i j}\left(y_{j}(t-\sigma(t))\right)\right] g_{i}\left(x_{i}\left(t-\sigma_{2}(t)\right)\right) .
\end{aligned}
$$

Remark 2. In this paper, the core of finite-time stability is that the system converges to a stable condition within a finite time. At present, the research on finite-time stability of the MBAMNNs with probabilistic time-varying delays and stochastic perturbation is very few. Thus, the proposed model is more general than some exciting results. A lot of models about stability of MBAMNNs are special cases of our considered model [31 34]. Therefore, the obtained results are much general and practical.

The corresponding response system can be described as

$$
\begin{aligned}
d \hat{x}_{i}(t) & \in\left[-a_{i} \hat{x}_{i}(t-\tau)+\sum_{j=1}^{m} c o\left[b_{j i}\left(\hat{x}_{i}(t)\right)\right] f_{j}\left(\hat{y}_{j}(t)\right)\right. \\
& +\delta(t) \sum_{j=1}^{m} c o\left[c_{j i}\left(\hat{x}_{i}(t-\tau(t))\right)\right] f_{j}\left(\hat{y}_{j}\left(t-\tau_{1}(t)\right)\right) \\
& \left.+(1-\delta(t)) \sum_{j=1}^{m} c o\left[c_{j i}\left(\hat{x}_{i}(t-\tau(t))\right)\right] f_{j}\left(\hat{y}_{j}\left(t-\tau_{2}(t)\right)\right)+u_{i}(t)\right] d t \\
& +\sum_{j=1}^{m} \beta_{j i}\left(t, e_{j}(t), e_{j}\left(t-\tau_{1}(t)\right)\right) d \omega_{j}(t)
\end{aligned}
$$


$+\sum_{j=1}^{m} \beta_{j 1}\left(t, e_{j}(t) \cdot e_{j}\left(t-\tau_{2}(t)\right)\right) d \omega_{j}(t)$

$d \hat{y}_{j}(t) \in\left[-d_{j} \hat{y}_{j}(t-\sigma)+\sum_{i=1}^{n} \operatorname{col}\left[q_{i j}\left(\hat{y}_{j}(t)\right)\right] g_{i}\left(\tilde{x}_{i}(t)\right)\right.$

$$
\begin{aligned}
& +\rho(t) \sum_{i=1}^{n} \operatorname{co}\left[p_{i j}\left(\hat{y}_{j}(t-\sigma(t))\right)\right] g_{i}\left(\hat{x}_{i}\left(t-\sigma_{1}(t)\right)\right) \\
& \left.+(1-\rho(t)) \sum_{i=1}^{n} \operatorname{co}\left[p_{i j}\left(\hat{y}_{j}(t-\sigma(t))\right)\right] g_{i}\left(\hat{x}_{i}\left(t-\sigma_{2}(t)\right)\right)+u_{j}(t)\right] d t \\
& +\sum_{i=1}^{n} \beta_{i j}\left(t, e_{i}(t), e_{i}\left(t-\sigma_{1}(t)\right)\right) d \omega_{i}(t) \\
& +\sum_{i=1}^{n} \beta_{i j}\left(t, e_{i}(t), e_{i}\left(t-\sigma_{2}(t)\right)\right) d \omega_{i}(t),
\end{aligned}
$$

where

$b_{j i}\left(x_{i}(t)\right)=\left\{\begin{aligned} \hat{b}_{j i}, & \left|x_{i}(t)\right|<\bar{w}_{i}, \\ \operatorname{co}\left[\underline{b}_{j i}, \bar{b}_{j i}\right], & \left|x_{i}(t)\right|=\bar{\varpi}_{i}, \\ \check{b}_{j i}, & \left|x_{i}(t)\right|>\varpi_{i},\end{aligned}\right.$

$b_{j i}\left(\hat{x}_{i}(t)\right)=\left\{\begin{aligned} \hat{b}_{j i}, & \left|\hat{x}_{i}(t)\right|<\varpi_{i}, \\ \operatorname{co}\left[\underline{b}_{j i}, \bar{b}_{j i}\right], & \left|\hat{x}_{i}(t)\right|=\varpi_{i}, \\ \breve{b}_{j i}, & \left|\hat{x}_{i}(t)\right|>\varpi_{i},\end{aligned}\right.$

$c_{j i}\left(x_{i}(t-\tau(t))\right)=\left\{\begin{aligned} \hat{\boldsymbol{c}}_{j i}, & \left|x_{i}(t-\tau(t))\right|<\varpi_{i}, \\ \operatorname{co}\left[\underline{c}_{j i}, \bar{c}_{j i}\right], & \left|x_{i}(t-\tau(t))\right|=\varpi_{i}, \\ \tilde{c}_{j i}, & \left|x_{i}(t-\tau(t))\right|>\varpi_{i},\end{aligned}\right.$

$c_{j i}\left(\hat{x}_{i}(t-\tau(t))\right)=\left\{\begin{aligned} \hat{c}_{j i}, & \left|\hat{x}_{i}(t-\tau(t))\right|<\varpi_{i}, \\ c o\left[c_{j i}, \bar{c}_{j i}\right], & \left|\hat{x}_{i}(t-\tau(t))\right|=\varpi_{i}, \\ \tilde{c}_{j i}, & \left|\hat{x}_{i}(t-\tau(t))\right|>\varpi_{i},\end{aligned}\right.$

$q_{i j}\left(y_{j}(t)\right)=\left\{\begin{aligned} \hat{q}_{i j}, & \left|y_{j}(t)\right|<\vartheta_{j}, \\ \operatorname{co}\left[\underline{q}_{i j}, \bar{q}_{i j}\right], & \left|y_{j}(t)\right|=\vartheta_{j}, \\ \tilde{q}_{i j}, & \left|y_{j}(t)\right|>\vartheta_{j},\end{aligned}\right.$

$q_{i j}\left(\hat{y}_{j}(t)\right)=\left\{\begin{aligned} \hat{q}_{i j}, & \left|\hat{y}_{j}(t)\right|<\vartheta_{j}, \\ \operatorname{co}\left[\underline{q}_{i j}, \bar{q}_{i j}\right], & \left|\hat{y}_{j}(t)\right|=\vartheta_{j}, \\ \tilde{q}_{i j}, & \left|\hat{y}_{j}(t)\right|>\vartheta_{j},\end{aligned}\right.$

$p_{i j}\left(y_{j}(t-\sigma(t))\right)=\left\{\begin{aligned} \hat{p}_{i j}, & \left|y_{j}(t-\sigma(t))\right|<\vartheta_{j}, \\ c o\left[\underline{p}_{i j}, \vec{p}_{i j}\right], & \left|y_{j}(t-\sigma(t))\right|=\vartheta_{j}, \\ \tilde{p}_{i j}, & \left|y_{j}(t-\sigma(t))\right|>\vartheta_{j} .\end{aligned}\right.$

$p_{i j}\left(\hat{y}_{j}(t-\sigma(t))\right)=\left\{\begin{aligned} \hat{p}_{i j}, & \left|\hat{y}_{j}(t-\sigma(t))\right|<\vartheta_{j}, \\ c o\left[\underline{p}_{i j}, \bar{p}_{i j}\right], & \left|\hat{y}_{j}(t-\sigma(t))\right|=\vartheta_{j}, \\ \tilde{p}_{i j}, & \left|\hat{y}_{j}(t-\sigma(t))\right|>\vartheta_{j},\end{aligned}\right.$

and

$\bar{b}_{j i}=\max \left\{\hat{b}_{j i}, \check{b}_{j i}\right\}, \quad \underline{b}_{j i}=\min \left\{\hat{b}_{j i}, \check{b}_{j i}\right\}$,

$\bar{c}_{j i}=\max \left\{\hat{c}_{j i}, \check{c}_{j i}\right\}, \quad \underline{c}_{j i}=\min \left\{\hat{c}_{j i}, \check{c}_{j i}\right\}$,

$\bar{q}_{i j}=\max \left\{\hat{q}_{i j}, \check{q}_{i j}\right\}, \quad \underline{q}_{i j}=\min \left\{\hat{q}_{i j}, \check{q}_{i j}\right\}$,

$\bar{p}_{i j}=\max \left\{\hat{p}_{i j}, \check{p}_{i j}\right\}, \quad \underline{p}_{i j}=\min \left\{\hat{p}_{i j}, \check{p}_{i j}\right\}$.

Let $e_{i}(t)$ and $e_{j}(t)$ denote the error variables, where $e_{i}(t)=$ $x_{i}(t)+\hat{x}_{i}(t)$ and $e_{j}(t)=y_{j}(t)+\hat{y}_{j}(t)$. From the Definitions 1 to 2 , we obtain the error systems as follows

$d e_{i}(t) \in\left[-a_{i} e_{i}(t-\tau)\right.$

$$
\begin{aligned}
& +\sum_{j=1}^{m}\left[\operatorname{co}\left[b_{j i}\left(x_{i}(t)\right)\right] f_{j}\left(y_{j}(t)\right)+\operatorname{co}\left[b_{j i}\left(\hat{x}_{i}(t)\right)\right] f_{j}\left(\hat{y}_{j}(t)\right)\right] \\
& +\delta(t) \sum_{j=1}^{m}\left[\operatorname{co}\left[c_{j i}\left(x_{i}(t-\tau(t))\right)\right] f_{j}\left(y_{j}\left(t-\tau_{1}(t)\right)\right)\right. \\
& \left.+\operatorname{co}\left[c_{j i}\left(\hat{x}_{i}(t-\tau(t))\right)\right] f_{j}\left(\hat{y}_{j}\left(t-\tau_{1}(t)\right)\right)\right] \\
& +(1-\delta(t)) \sum_{j=1}^{m}\left[\operatorname{co}\left[c_{j i}\left(x_{i}(t-\tau(t))\right)\right] f_{j}\left(y_{j}\left(t-\tau_{2}(t)\right)\right)\right. \\
& \left.\left.+c o\left[c_{j i}\left(\hat{x}_{i}(t-\tau(t))\right)\right] f_{j}\left(\hat{y}_{j}\left(t-\tau_{2}(t)\right)\right)\right]+u_{i}(t)\right] d t \\
& +\sum_{j=1}^{m} \beta_{j i}\left(t, e_{j}(t), e_{j}\left(t-\tau_{1}(t)\right)\right) d \omega_{j}(t) \\
& +\sum_{j=1}^{m} \beta_{j i}\left(t, e_{j}(t), e_{j}\left(t-\tau_{2}(t)\right)\right) d \omega_{j}(t)
\end{aligned}
$$

$$
\begin{aligned}
d e_{j}(t) \in & {\left[-d_{j} e_{j}(t-\sigma)\right.} \\
& +\sum_{i=1}^{n}\left[\operatorname{co}\left[q_{i j}\left(y_{j}(t)\right)\right] g_{i}\left(x_{i}(t)\right)+\operatorname{co}\left[q_{i j}\left(\hat{y}_{j}(t)\right)\right] g_{i}\left(\hat{x}_{i}(t)\right)\right] \\
& +\rho(t) \sum_{i=1}^{n}\left[\operatorname{co}\left[p_{i j}\left(y_{j}(t-\sigma(t))\right)\right] g_{i}\left(x_{i}\left(t-\sigma_{1}(t)\right)\right)\right. \\
& \left.+\operatorname{co}\left[p_{i j}\left(\hat{y}_{j}(t-\sigma(t))\right)\right] g_{i}\left(\hat{x}_{i}\left(t-\sigma_{1}(t)\right)\right)\right] \\
& +(1-\rho(t)) \sum_{i=1}^{n}\left[\operatorname{co}\left[p_{i j}\left(\hat{y}_{j}(t-\sigma(t))\right)\right] g_{i}\left(\hat{x}_{i}\left(t-\sigma_{2}(t)\right)\right)\right. \\
& \left.\left.+\operatorname{co}\left[p_{i j}\left(y_{j}(t-\sigma(t))\right)\right] g_{i}\left(x_{i}\left(t-\sigma_{2}(t)\right)\right)\right]+u_{j}(t)\right] d t \\
& +\sum_{i=1}^{n} \beta_{i j}\left(t, e_{i}(t), e_{i}\left(t-\sigma_{1}(t)\right)\right) d \omega_{i}(t) \\
& +\sum_{i=1}^{n} \beta_{i j}\left(t, e_{i}(t), e_{i}\left(t-\sigma_{2}(t)\right)\right) d \omega_{i}(t) .
\end{aligned}
$$

Throughout this paper, we have the following Assumptions and Definitions.

Definition 1 [37]. Suppose $B \subset \Re^{n}$. Then $d \mapsto F(d)$ is called as a setvalued map defined on $B$, if for each point $d$ of $B$, there exists a corresponding nonempty set $F(d) \subset \mathfrak{R}^{n}$. A set-valued map $F$ with nonempty values is considered to be upper-semicontinuous at $d_{0} \in B$, if for any open set $N$ containing $F\left(d_{0}\right)$, there exists a neighborhood $K$ of $d_{0}$ such that $F(K) \subset N . F(d)$ is said to have a closed image if for each $d \in B, F(d)$ is closed.

Definition 2 [27]. For the system $\dot{x}(t)=f(x), x \in \mathbb{R}^{n}$, with discontinuous right-hand sides, a set-valued map is defined as

$F(t, x)=\bigcap_{\delta>0} \bigcap_{\mu(N)=0} \operatorname{co}[f(B(x, \delta) \backslash N)]$,

where $c o[E]$ is the closure of the convex hull of set $E, B(x, \delta)=\{y$ : $\|y-x\| \leq \delta\}$ and $\mu(N)$ is Lebesgue measure of set $N$. A solution in Filippov's sense of Cauchy problem for this system with initial condition $x(0)=x_{0}$ is an absolutely continuous function $x(t)$, which satisfies $x(0)=x_{0}$ and differential inclusion $\dot{x}(t) \in F(t, x)$.

Definition 3 [38]. The trivial solution of system (1) or (2) is called stochastically finite-time stable, if there exist constants $t_{1}>0$ and $t_{2}>0, t_{1}$ and $t_{2}$ depend on the 
value of state variables $e_{i}(0)=\left(e_{1}(0), e_{2}(0), \ldots, e_{n}(0)\right)^{\mathrm{T}}$ and $e_{j}(0)=\left(e_{1}(0), e_{2}(0) \ldots, e_{m}(0)\right)^{\mathrm{T}}$, such that the following inequality holds:

$\sum_{i=1}^{n} \mathbb{E}\left\|e_{i}(t)\right\|^{2}=0 . \quad \sum_{j=1}^{m} \mathbb{E}\left\|e_{j}(t)\right\|^{2}=0$.

and

$\mathbb{E}\left\|e_{i}(t)\right\|^{2} \equiv 0, \quad \mathbb{E}\left\|e_{j}(t)\right\|^{2} \equiv 0$

under any initial condition.

$\phi(s)=\left(\phi_{1}(s), \phi_{2}(s), \ldots, \phi_{n}(s)\right)^{\mathrm{T}}$,

$\gamma(s)=\left(\gamma_{1}(s), \gamma_{2}(s), \ldots, \gamma_{n}(s)\right)^{\mathrm{T}} \in \mathcal{C}\left([-v, 0], \mathbb{R}^{n}\right)$,

which denotes the Banach space of all continuous functions mapping $[-\nu, 0]$ into $\mathbb{R}^{n}$ and $\nu=\max \left\{\tau, \tau_{2}\right\}$.

$\varphi(s)=\left(\varphi_{1}(s), \varphi_{2}(s), \ldots, \varphi_{m}(s)\right)^{\mathrm{T}}$,

$\psi(s)=\left(\psi_{1}(s), \psi_{2}(s), \ldots, \psi_{m}(s)\right)^{\mathrm{T}} \in \mathcal{C}\left([-5,0], \mathbb{R}^{m}\right)$.

which denotes the Banach space of all continuous functions mapping $[-\zeta, 0]$ into $\mathbb{R}^{m}$ and $\zeta=\max \left\{\sigma, \sigma_{2}\right\}$.

Definition 4 [29]. Systems (1) and (2) are said to be stochastically finite-time stable for suitable feedback controller $u_{i}(t)$ and $u_{i}(t)$, there are exist constants $T_{x}>0$ and $T_{y}>0$ such that

$\lim _{t \rightarrow T_{x}}\left\|e_{i}(t)\right\|=0, i=1,2, \ldots, n, \lim _{t \rightarrow T_{y}}\left\|e_{j}(t)\right\|=0, j=1,2, \ldots, m$.

And $\left\|e_{i}(t)\right\|=0$ for $t>T_{x},\left\|e_{j}(t)\right\|=0$ for $t>T_{y}$, where $T_{x}$ and $T_{y}$ are functions about the initial state vector values $e_{i}(t)=\gamma_{i}(t)$ for $t \in[-v, 0]$ and $e_{j}(t)=\psi_{j}(t)$ for $t \in[-5,0]$. The functions $T_{x}$ and $T_{y}$ are named the setting-time functions and their values are called the setting times.

Assumption 1 [16]. There exist constants $R_{1} \geq 0$ and $R_{2} \geq 0$, such that

$\operatorname{Trace}\left[\sigma^{\mathrm{T}}(t, x(t), x(t-\xi(t))) \sigma(t, x(t), x(t-\xi(t)))\right]$

$\leq x^{T}(t) R_{1} x(t)+x^{\mathrm{T}}(t-\xi(t)) R_{2} x(t-\xi(t))$.

For the stochastic system [38]:

$d y(t)=g(t, y(t)) d t+\sigma(t, y(t)) d \omega(t)$,

where $\omega(t)$ is the Brownian motion and it is truely $\mathbb{E} \omega(t)=0$. $\mathscr{L}$ is the operator designed as following:

$$
\begin{aligned}
\mathscr{L} V(t, y) & =V_{t}(t, y)+V_{y} g(t, y) \\
& +\frac{1}{2} \operatorname{Trace}\left[\sigma^{\mathrm{T}}(t, y(t)) V_{y y} \sigma(t, y(t))\right],
\end{aligned}
$$

where

$V_{t}(t, y)=\frac{\partial V_{t}(t, y)}{\partial t}$

$V_{y y}(t, y)=\left(\frac{\partial^{2} V_{t}(t, y)}{\partial y_{i} \partial y_{j}}\right)$,

$V_{y}(t, y)=\left(\frac{\partial V_{t}(t, y)}{\partial y_{1}}, \frac{\partial V_{t}(t, y)}{\partial y_{2}}, \ldots, \frac{\partial V_{t}(t, y)}{\partial y_{n}}\right)^{\mathrm{T}}$.

Assumption 2. For $i=1, \ldots, n, j=1, \ldots, m$,

$$
\begin{aligned}
& {\left[\underline{b}_{j i}, \bar{b}_{j i}\right] f_{j}\left(y_{j}(t)\right)+\left[\underline{b}_{j i}, \bar{b}_{j i}\right] f_{j}\left(\hat{y}_{j}(t)\right) \subseteq\left[\underline{b}_{j i}, \bar{b}_{j i}\right]\left(f_{j}\left(y_{j}(t)\right)+f_{j}\left(\hat{y}_{j}(t)\right)\right),} \\
& {\left[\underline{c}_{j i}, \bar{c}_{j i}\right] f_{j}\left(y_{j}(t)\right)+\left[\underline{c}_{j i}, \bar{c}_{j i}\right] f_{j}\left(\hat{y}_{j}(t)\right) \subseteq\left[\underline{c}_{j i}, \bar{c}_{j i}\right]\left(f_{j}\left(y_{j}(t)\right)+f_{j}\left(\hat{y}_{j}(t)\right)\right),} \\
& {\left[\underline{p}_{i j}, \bar{p}_{i j}\right] g_{i}\left(x_{i}(t)\right)+\left[\underline{p}_{i j}, \bar{p}_{i j}\right] g_{i}\left(\hat{x}_{i}(t)\right) \subseteq\left[\underline{p}_{i j}, \bar{p}_{i j}\right]\left(g_{i}\left(x_{i}(t)\right)+g_{i}\left(\hat{x}_{i}(t)\right)\right),} \\
& {\left[\underline{q}_{i j}, \bar{q}_{i j}\right] g_{i}\left(x_{i}(t)\right)+\left[\underline{q}_{i j}, \bar{q}_{i j}\right] g_{i}\left(\hat{x}_{i}(t)\right) \subseteq\left[\underline{q}_{i j}, \bar{q}_{i j}\right]\left(g_{i}\left(x_{i}(t)\right)+g_{i}\left(\hat{x}_{i}(t)\right)\right) .}
\end{aligned}
$$

Remark 3. Some papers [55-57] of stability or synchronization of MNNs are derived from the assumption

$$
\operatorname{co}\left[\underline{a}_{i j}, \bar{a}_{i j}\right] f_{j}\left(y_{j}(t)\right)-\operatorname{co}\left[\underline{a}_{i j}, \bar{a}_{i j}\right] f_{j}\left(x_{j}(t)\right) \subseteq \operatorname{co}\left[\underline{a}_{i j}, \bar{a}_{i j}\right]\left(f_{j}\left(y_{j}(t)\right)\right.
$$

$$
\left.-f_{j}\left(x_{j}(t)\right)\right)
$$

but it should be mentioned that this assumption has been proved unreasonable in [36]. During the proof of Theorems 1 and 2 in the present paper, by making full use of Assumption 3 and activation functions, we put the knotty terms together to design the suitable error systems then get the inequalities Eqs. (10) and (11), successfully avoiding the assumption problem.

Assumption 3 [38]. The activation functions $f_{j}(\cdot)(j=1.2 \ldots \ldots m)$ and $g_{i}(\cdot)(i=1.2 \ldots n)$ are odd, bounded and globally Lipschitz continuous in $\mathbb{R}$, namely, there exist constants $\underline{L}_{x}, \bar{L}_{x}, \underline{L}_{y}$ and $\bar{L}_{y}$ for all $s_{1}, s_{2} \in \mathbb{R}, s_{1} \neq s_{2}$ such that

$$
\begin{aligned}
& \underline{L}_{x} \leq \frac{f_{j}\left(s_{1}\right)-f_{j}\left(s_{2}\right)}{s_{1}-s_{2}} \leq \bar{L}_{x}, \\
& \underline{L}_{y} \leq \frac{g_{i}\left(s_{1}\right)-g_{i}\left(s_{2}\right)}{s_{1}-s_{2}} \leq \bar{L}_{y},
\end{aligned}
$$

that the constants $\underline{L}_{x}, \bar{L}_{x}, \underline{L}_{y}$ and $\bar{L}_{y}$ can be positive numbers, negative numbers or zero.

Remark 3. According to Assumptions 2 and 3 , the activations functions $f_{j}(t)$ and $g_{i}(t)$ are odd functions. Then for $e_{i}(t) \neq 0$ and $e_{j}(t) \neq 0$, we get $F_{j}\left(e_{j}(t)\right)$ and $G_{i}\left(e_{i}(t)\right)$ possess the following properties [25]:

$$
\begin{aligned}
& \underline{L}_{x} e_{j}(t) \leq F_{j}\left(e_{j}(t)\right) \leq \bar{L}_{x} e_{j}(t), \\
& \underline{L}_{y} e_{i}(t) \leq G_{i}\left(e_{i}(t)\right) \leq \bar{L}_{y} e_{i}(t) .
\end{aligned}
$$

and

$F_{j}(0)=f_{j}\left(y_{j}(t)\right)+f_{j}\left(-y_{j}(t)\right), \quad G_{i}(0)=g_{i}\left(x_{i}(t)\right)+g_{i}\left(-x_{i}(t)\right)$.

Therefore, the error systems admits zero solutions $e_{\bar{i}}(t) \equiv 0$ and $e_{j}(t) \equiv 0$.

Lemma 1 [24]. Give any real matrices $X, Z, P$ of appropriate dimensions and scalars $\epsilon_{0}>0, P>0$, the following inequality holds:

$$
X^{\mathrm{T}} Z+Z^{\mathrm{T}} X \leq \epsilon_{0} X^{\mathrm{T}} P X+\epsilon_{0}^{-1} Z^{\mathrm{T}} P Z^{-1} Z .
$$

In particular, if $X$ and $Z$ are vectors, $X^{\mathrm{T}} Z \leq \frac{1}{2}\left(X^{\mathrm{T}} X+Z^{\mathrm{T}} Z\right)$.

Lemma 2 [38]. If there is a continuous, positive definite function $V(t)$ satisfied following differential inequality:

$$
\dot{V}(t)+\alpha V^{\eta}(t) \leq 0 . \forall t \geq t_{0}, V\left(t_{0}\right) \geq 0,
$$

where $\alpha>0,0<\eta<1$ are two constants. Then, for any provided $t_{0}$, $V(t)$ satisfies the following differential inequality:

$$
V^{1-\eta}(t) \leq V^{1-\eta}\left(t_{0}\right)-\alpha(1-\eta)\left(t-t_{0}\right), t_{0} \leq t \leq t_{1},
$$

and

$$
V(t) \equiv 0, \forall t \geq t_{1},
$$

with $t_{1}$ defined by

$$
t_{1}=t_{0}+\frac{V^{1-\eta}\left(t_{0}\right)}{\alpha(1-\eta)} \text {. }
$$

Lemma 3 [40]. Let $x_{1}, x_{2}, \ldots, x_{n} \in R^{n}$ are any vectors and $0<q<2$ is a real number satisfying:

$$
\left\|x_{1}\right\|^{q}+\left\|x_{2}\right\|^{q}+\cdots+\left\|x_{n}\right\|^{q} \geq\left(\left\|x_{1}\right\|^{2}+\left\|x_{2}\right\|^{2}+\cdots+\left\|x_{n}\right\|^{2}\right)^{q / 2} .
$$

Remark 4. Comparing with the exciting literatures for researching the finite synchronization of MBAMNNs [40], the considered systems contained not only non-delay, discrete time-varying delays but also distributed delays and leakage delays. Therefore, the obtained results are much practical and reasonable.

\section{Main results}

In this section, the finite-time anti-synchronization of MBAMNNs with probabilistic time-varying delays and stochastic perturbations are investigated. 
Theorem 1. Under Assumptions 1-3, the error system (5) of the stochastic MBAMNNs with probabilistic time-varying will globally stable in finite time via the following controllers

$u_{i}(t)=-\eta_{x} e_{i}(t)-k_{x} \operatorname{sign}\left(e_{i}(t)\right)\left|e_{i}(t)\right|^{\alpha}$,

$u_{j}(t)=-\eta_{y} e_{j}(t)-k_{y} \operatorname{sign}\left(e_{j}(t)\right)\left|e_{j}(t)\right|^{\alpha}$,

if there exists the gain constants $\eta_{x}, \eta_{y}$ and $k_{x}, k_{y}$ such that

$\left\{\begin{array}{l}\sum_{i=1}^{n}\left[\left|a_{i}\right|-\sum_{j=1}^{m}\left|\bar{b}_{j i}\right| \bar{L}_{x}-\sum_{j=1}^{m}\left|\bar{c}_{j i}\right| \bar{L}_{x}-R_{1}-R_{3}\right. \\ \left.+2 \eta_{x}\right]-\sum_{i=1}^{m} \sum_{j=1}^{n}\left|\bar{b}_{i j}\right| \bar{L}_{x} \geq 0, \\ \left|\bar{c}_{j i}\right| \bar{L}_{x} \delta_{0}+\frac{1}{m} R_{2} \leq 0 .\end{array}\right.$

$\left|\bar{c}_{j i}\right| \bar{L}_{x}\left(1-\delta_{0}\right)+\frac{1}{m} R_{4} \leq 0$.

and

$$
\left\{\begin{array}{l}
\sum_{j=1}^{m}\left[\left|d_{j}\right|-\sum_{i=1}^{n}\left|\bar{q}_{i j}\right| \bar{L}_{y}-\sum_{i=1}^{n}\left|\bar{p}_{i j}\right| \bar{L}_{y}-P_{1}-P_{3}\right. \\
\left.+2 \eta_{y}\right]-\sum_{i=1}^{m} \sum_{j=1}^{n}\left|\bar{q}_{j i}\right| \bar{L}_{y} \geq 0, \\
\left|\bar{p}_{i j}\right| \bar{L}_{y} \rho_{0}+\frac{1}{n} P_{2} \leq 0, \\
\left|\bar{p}_{i j}\right| \bar{L}_{y}\left(1-\rho_{0}\right)+\frac{1}{n} P_{4} \leq 0 .
\end{array}\right.
$$

where $R_{1}, R_{2}, R_{3}, R_{4}$ and $P_{1}, P_{2}, P_{3}, P_{4}$ are all positive constants determined in the later. Then, the synchronization time is estimated by

$$
\begin{aligned}
& T_{x}=\frac{V^{\frac{1-\alpha}{2}}(0)}{k_{x}(1-\alpha)}=\frac{\left\|e_{i}(0)\right\|^{1-\alpha}}{k_{x}(1-\alpha)}, \\
& T_{y}=\frac{V^{\frac{1-\alpha}{2}}(0)}{k_{y}(1-\alpha)}=\frac{\left\|e_{j}(0)\right\|^{1-\alpha}}{k_{y}(1-\alpha)} .
\end{aligned}
$$

Proof. Construct the following Lyapunov function

$$
V\left(t, e_{i}(t), e_{j}(t)\right)=\sum_{i=1}^{n} e_{i}^{T}(t) e_{i}(t)+\sum_{j=1}^{m} e_{j}^{T}(t) e_{j}(t)
$$

Let

$V_{1}(t)=\sum_{i=1}^{n} e_{i}^{T}(t) e_{i}(t), V_{2}(t)=\sum_{j=1}^{m} e_{j}^{T}(t) e_{j}(t)$.

Then

$V\left(t . e_{i}(t), e_{j}(t)\right)=V_{1}(t)+V_{2}(t)$.

$\mathbb{E}\left\{\mathcal{L} V\left(t, e_{i}(t), e_{j}(t)\right)\right\}=\mathbb{E}\left\{\mathcal{L} V_{1}(t)\right\}+\mathbb{E}\left\{\mathcal{L} V_{2}(t)\right\}$.

And

$\mathbb{E}\left\{\mathcal{L} V_{1}(t)\right\}=2 \sum_{i=1}^{n} e_{i}(t) \dot{e}_{i}(t)$,

$\mathbb{E}\left\{\mathcal{L} V_{2}(t)\right\}=2 \sum_{j=1}^{m} e_{j}(t) \dot{e}_{j}(t)$

Under the Assumption 2, the error systems be obtained as

$$
\begin{aligned}
d e_{i}(t) \in & {\left[-a_{i} e_{j}(t-\tau)+\sum_{j=1}^{m}\left[\left[\underline{b}_{j i}, \bar{b}_{j i}\right]\left(f_{j}\left(y_{j}(t)\right)+f_{j}\left(\hat{y}_{j}(t)\right)\right)\right]\right.} \\
& +\delta(t) \sum_{j=1}^{m}\left[\left[\underline{c}_{j i}, \bar{c}_{j i}\right]\left(f_{j}\left(y_{j}\left(t-\tau_{1}(t)\right)\right)+f_{j}\left(\hat{y}_{j}\left(t-\tau_{1}(t)\right)\right)\right)\right] \\
& +(1-\delta(t)) \sum_{j=1}^{m}\left[[ \underline { c } _ { j i } , \overline { c } _ { j i } ] \left(f_{j}\left(y_{j}\left(t-\tau_{2}(t)\right)\right)\right.\right. \\
& \left.\left.\left.+f_{j}\left(\hat{y}_{j}\left(t-\tau_{2}(t)\right)\right)\right)\right]+u_{i}(t)\right] d t \\
& +\sum_{j=1}^{m} \beta_{j i}\left(t, e_{j}(t), e_{j}(t-\tau(t))\right) d \omega_{j}(t) \\
& +\sum_{j=1}^{m} \beta_{j i}\left(t, e_{j}(t), e_{j}\left(t-\tau_{2}(t)\right)\right) d \omega_{j}(t),
\end{aligned}
$$

$$
\begin{aligned}
d e_{j}(t) \in & {\left[-d_{j} e_{j}(t-\sigma)+\sum_{i=1}^{n}\left[\left[\underline{q}_{i j}, \bar{q}_{i j}\right]\left(g_{i}\left(x_{i}(t)\right)+g_{i}\left(\hat{x}_{i}(t)\right)\right)\right]\right.} \\
& +\rho(t) \sum_{i=1}^{n}\left[\left[\underline{p}_{i j}, \bar{p}_{i j}\right]\left(g_{i}\left(x_{i}\left(t-\sigma_{1}(t)\right)\right)+g_{i}\left(\hat{x}_{i}\left(t-\sigma_{1}(t)\right)\right)\right)\right] \\
& +(1-\rho(t)) \sum_{i=1}^{n}\left[[ \underline { p } _ { i j } \cdot \overline { p } _ { i j } ] \left(g_{i}\left(\hat{x}_{i}\left(t-\sigma_{2}(t)\right)\right)\right.\right. \\
& \left.\left.\left.+g_{i}\left(x_{i}\left(t-\sigma_{2}(t)\right)\right)\right)\right]+u_{j}(t)\right] d t \\
& +\sum_{i=1}^{n} \beta_{i j}\left(t, e_{i}(t), e_{i}(t-\sigma(t))\right) d \omega_{i}(t) \\
& +\sum_{i=1}^{n} \beta_{i j}\left(t, e_{i}(t), e_{i}\left(t-\sigma_{2}(t)\right)\right) d \omega_{i}(t) .
\end{aligned}
$$

Then we get

$$
\begin{aligned}
d e_{i}(t) \leq & {\left[\delta(t) \sum_{j=1}^{m}\left[\bar{c}_{j i}\left[f_{j}\left(y_{j}\left(t-\tau_{1}(t)\right)\right)+f_{j}\left(\hat{y}_{j}\left(t-\tau_{1}(t)\right)\right)\right]\right]\right.} \\
& -a_{i} e_{i}(t-\tau)+\sum_{j=1}^{m}\left[\bar{b}_{j i}\left(f_{j}\left(y_{j}(t)\right)+f_{j}\left(\hat{y}_{j}(t)\right)\right)\right] \\
& +(1-\delta(t)) \sum_{j=1}^{m}\left[\overline { c } _ { j i } \left[f_{j}\left(y_{j}\left(t-\tau_{2}(t)\right)\right)\right.\right. \\
& \left.\left.\left.+f_{j}\left(\hat{y}_{j}\left(t-\tau_{2}(t)\right)\right)\right]\right]+u_{i}(t)\right] d t \\
& +\sum_{j=1}^{m} \beta_{j i}\left(t, e_{j}(t), e_{j}(t-\tau(t))\right) d \omega_{j}(t) \\
& +\sum_{j=1}^{m} \beta_{j i}\left(t, e_{j}(t), e_{j}\left(t-\tau_{2}(t)\right)\right) d \omega_{j}(t)
\end{aligned}
$$

And

$$
\begin{aligned}
d e_{i}(t) \leq & {\left[-a_{i} e_{i}(t-\tau)+\sum_{j=1}^{m} \bar{b}_{j i} F_{j}\left(e_{j}(t)\right)\right.} \\
& +\delta(t) \sum_{j=1}^{m} \bar{c}_{j i} F_{j}\left(e_{j}\left(t-\tau_{1}(t)\right)\right) \\
& \left.+(1-\delta(t)) \sum_{j=1}^{m} \bar{c}_{j i} F_{j}\left(e_{j}\left(t-\tau_{2}(t)\right)\right)+u_{i}(t)\right] d t \\
& +\sum_{j=1}^{m} \beta_{j i}\left(t, e_{j}(t), e_{j}(t-\tau(t))\right) d \omega_{j}(t) \\
& +\sum_{j=1}^{m} \beta_{j i}\left(t, e_{j}(t), e_{j}\left(t-\tau_{2}(t)\right)\right) d \omega_{j}(t),
\end{aligned}
$$

where $\quad F_{j}\left(e_{j}(t)\right)=f_{j}\left(y_{j}(t)\right)+f_{j}\left(\hat{y}_{j}(t)\right), \quad F_{j}\left(e_{j}\left(t-\tau_{1}(t)\right)\right)=$ $f_{j}\left(y_{j}\left(t-\tau_{1}(t)\right)\right)+f_{j}\left(\hat{y}_{j}\left(t-\tau_{1}(t)\right)\right), \quad F_{j}\left(e_{j}\left(t-\tau_{2}(t)\right)\right)=f_{j}\left(y_{j}(t-\right.$ $\left.\left.\tau_{2}(t)\right)\right)+f_{j}\left(\hat{y}_{j}\left(t-\tau_{2}(t)\right)\right)$.

Then we have

$$
\begin{aligned}
\mathbb{E}\left\{\mathcal{L} V_{1}(t)\right\}= & 2 \sum_{i=1}^{n} e_{i}(t)\left[-a_{i} e_{i}(t-\tau)+\sum_{j=1}^{m} \bar{b}_{j i} F_{j} e_{j}(t)+u_{i}(t)\right. \\
& \left.+\delta_{0} \sum_{j=1}^{m} \bar{c}_{j i} F_{j} e_{j}\left(t-\tau_{1}(t)\right)+\left(1-\delta_{0}\right) \sum_{j=1}^{m} \bar{c}_{j i} F_{j} e_{j}\left(t-\tau_{2}(t)\right)\right] \\
& +\sum_{j=1}^{m} \operatorname{Trace}\left[\rho_{j i}^{\mathrm{T}}\left(t, y(t), y\left(t-\tau_{1}(t)\right)\right) \rho_{j i}(t, y(t),\right.
\end{aligned}
$$




$$
\begin{aligned}
& \left.\left.y\left(t-\tau_{1}(t)\right)\right)\right]+\sum_{j=1}^{m} \operatorname{Trace}\left[\rho_{j i}^{\mathrm{T}}\left(t, y(t), y\left(t-\tau_{2}(t)\right)\right)\right. \\
& \left.\rho_{j l}\left(t, y(t), y\left(t-\tau_{2}(t)\right)\right)\right] .
\end{aligned}
$$

According to the Assumption 1 and controller (9), we get

$$
\begin{aligned}
\mathbb{E}\left\{\mathcal{L} V_{1}(t)\right\} \leq & -2 \sum_{i=1}^{n}\left|a_{i}\right| e_{i}(t) e_{i}(t-\tau)+2 \sum_{i=1}^{n} \sum_{j=1}^{m}\left|\bar{b}_{j i}\right| e_{i}(t) F_{j}\left(e_{j}(t)\right) \\
& +2 \sum_{i=1}^{n} \sum_{j=1}^{m} \delta_{0}\left|\bar{c}_{j j}\right| e_{i}(t) F_{j}\left(e_{j}\left(t-\tau_{1}(t)\right)\right)-2 \sum_{i=1}^{n} \eta_{x} e_{i}^{2}(t) \\
& +2 \sum_{i=1}^{n} \sum_{j=1}^{m}\left(1-\delta_{0}\right)\left|\bar{c}_{j i}\right| e_{i}(t) F_{j}\left(e_{j}\left(t-\tau_{2}(t)\right)\right) \\
& -2 \sum_{i=1}^{n} k_{x} e_{i}(t) \operatorname{sign}\left(e_{i}(t)\right)\left|e_{i}(t)\right|^{\alpha}+\sum_{j=1}^{m} e_{j}^{T}(t) R_{1} e_{j}(t) \\
& +\sum_{j=1}^{m} e_{j}^{T}\left(t-\tau_{1}(t)\right) R_{2} e_{i}\left(t-\tau_{1}(t)\right)+\sum_{j=1}^{m} e_{j}^{T}(t) R_{3} e_{j}(t) \\
& +\sum_{j=1}^{m} e_{j}^{\mathrm{T}}\left(t-\tau_{2}(t)\right) R_{4} e_{j}\left(t-\tau_{2}(t)\right) .
\end{aligned}
$$

Based on the Assumption 3 and Lemma 1, we conclude

$$
\begin{aligned}
2 \sum_{i=1}^{n}\left|a_{i}\right| e_{i}(t) e_{i}(t-\tau) & \leq \sum_{i=1}^{n}\left|a_{i}\right|\left[e_{i}^{2}(t)+e_{i}^{2}(t-\tau)\right], \\
2 \sum_{i=1}^{n} \sum_{j=1}^{m}\left|\bar{b}_{j i}\right| e_{i}(t) F_{j} e_{j}(t) & \leq 2 \sum_{i=1}^{n} \sum_{j=1}^{m}\left|\bar{b}_{j i}\right| \bar{L}_{y} e_{i}(t) e_{j}(t) \\
& \leq \sum_{i=1}^{n} \sum_{j=1}^{m}\left|\bar{b}_{j i}\right| \bar{L}_{y}\left[e_{i}^{2}(t)+e_{j}^{2}(t)\right],
\end{aligned}
$$

$$
\begin{aligned}
& 2 \delta_{0} \sum_{i=1}^{n} \sum_{j=1}^{m}\left|\bar{c}_{j i}\right| e_{i}(t) F_{j} e_{j}\left(t-\tau_{1}(t)\right) \\
& \leq 2 \delta_{0} \sum_{i=1}^{n} \sum_{j=1}^{m}\left|\bar{c}_{j i}\right| \bar{L}_{y} e_{i}(t) e_{j}\left(t-\tau_{1}(t)\right) \\
& \leq \delta_{0} \sum_{i=1}^{n} \sum_{j=1}^{m}\left|\bar{c}_{j i}\right| \bar{L}_{y}\left[e_{i}^{2}(t)+e_{j}^{2}\left(t-\tau_{1}(t)\right)\right], \\
& 2\left(1-\delta_{0}\right) \sum_{i=1}^{n} \sum_{j=1}^{m}\left|\bar{c}_{j i}\right| e_{i}(t) F_{j} e_{j}\left(t-\tau_{2}(t)\right) \\
& \leq 2\left(1-\delta_{0}\right) \sum_{i=1}^{n} \sum_{j=1}^{m}\left|\bar{c}_{j i}\right| \bar{L}_{y} e_{i}(t) e_{j}\left(t-\tau_{2}(t)\right) \\
& \leq\left(1-\delta_{0}\right) \sum_{i=1}^{n} \sum_{j=1}^{m}\left|\bar{c}_{j i}\right| \bar{L}_{y}\left[e_{i}^{2}(t)+e_{j}^{2}\left(t-\tau_{2}(t)\right)\right],
\end{aligned}
$$

By Eqs. (18) and (19), we have

$$
\begin{aligned}
\mathbb{E}\left\{\mathcal{L} V_{1}(t)\right\} \leq & -\left\{\sum _ { i = 1 } ^ { n } \left[\left|a_{i}\right|-\sum_{j=1}^{m}\left|\bar{b}_{j i}\right| \bar{L}_{x}\right.\right. \\
& \left.\left.-\sum_{j=1}^{m}\left|\bar{c}_{j i}\right| \bar{L}_{x}-R_{1}-R_{3}+2 \eta_{x}\right]-\sum_{i=1}^{m} \sum_{j=1}^{n}\left|\bar{b}_{i j}\right| \bar{L}_{x}\right\} e_{i}^{2}(t) \\
& -\sum_{i=1}^{n}\left|a_{i}\right| e_{i}^{2}(t-\tau)+\sum_{i=1}^{n}\left[\sum_{j=1}^{m}\left|\bar{c}_{j i}\right| \bar{L}_{x} \delta_{0}+R_{2}\right] e_{j}^{2}\left(t-\tau_{1}(t)\right) \\
& +\sum_{i=1}^{n}\left[\sum_{j=1}^{m}\left|\bar{c}_{j i}\right| \bar{L}_{x}\left(1-\delta_{0}\right)+R_{\mathbf{4}}\right] e_{j}^{2}\left(t-\tau_{2}(t)\right)
\end{aligned}
$$

$$
-2 \sum_{i=1}^{n} k_{x} e_{i}(t) \operatorname{sign}\left(e_{i}(t)\right)\left|e_{i}(t)\right|^{\alpha} .
$$

Due to

$\sum_{i=1}^{n} k_{x} e_{i}(t) \operatorname{sign}\left(e_{i}(t)\right)\left|e_{i}(t)\right|^{\alpha}=\sum_{i=1}^{n} k_{x}\left|e_{i}(t)\right|^{\alpha+1}$,

From Lemma 3, We get

$\left(\sum_{i=1}^{n}\left|e_{i}(t)\right|^{\alpha+1}\right)^{\frac{1}{\alpha+1}}=\left(\sum_{i=1}^{n}\left|e_{i}(t)\right|^{2}\right)^{\frac{1}{2}}$

Hence

$\sum_{i=1}^{n}\left|e_{i}(t)\right|^{\alpha+1}=\left(\sum_{i=1}^{n}\left|e_{i}(t)\right|^{2}\right)^{\frac{\alpha+1}{2}}=\left(\sum_{i=1}^{n} e_{i}^{2}(t)\right)^{\frac{\alpha+1}{2}}$.

Then we conclude

$$
\begin{aligned}
& \mathbb{E}\left\{\mathcal{L} V_{1}(t)\right\} \leq-\left\{\sum _ { i = 1 } ^ { n } \left[\left|a_{i}\right|-\sum_{j=1}^{m}\left|\bar{b}_{j i}\right| \bar{L}_{x}\right.\right. \\
& \left.\left.-\sum_{j=1}^{m}\left|\bar{c}_{j i}\right| \bar{L}_{x}-R_{1}-R_{3}+2 \eta_{x}\right]-\sum_{i=1}^{m} \sum_{j=m}^{n}\left|\bar{b}_{i j}\right| \bar{L}_{x}\right\} e_{i}^{2}(t) \\
& \quad-\sum_{i=1}^{n}\left|a_{i}\right| e_{i}^{2}(t-\tau)+\sum_{i=1}^{n}\left[\sum_{j=1}^{m}\left|\bar{c}_{j i}\right| \bar{L}_{x} \delta_{0}+R_{2}\right] e_{j}^{2}\left(t-\tau_{1}(t)\right) \\
& +\sum_{i=1}^{n}\left[\sum_{j=1}^{n}\left|\bar{c}_{j i}\right| \bar{L}_{x}\left(1-\delta_{0}\right)+R_{4}\right] e_{j}^{2}\left(t-\tau_{2}(t)\right)-2 k_{x}\left(\sum_{i=1}^{n} e_{i}^{2}(t)\right)^{\frac{n+1}{2}} .
\end{aligned}
$$

By Theorem 1 and Lemma 3, we get

$$
\mathbb{E}\left\{\mathcal{L} V_{1}(t)\right\} \leq \mathbb{E}\left\{-2 k_{x}\left(\sum_{i=1}^{n} e_{i}^{2}(t)\right)^{\frac{\alpha+1}{2}}\right\}=-2 k_{x} \mathbb{E}\left\{V_{1}^{\frac{\alpha+1}{2}}(t)\right\}
$$

With the similar process of $\mathcal{L} V_{1}(t)$, we also get

$$
\mathbb{E}\left\{\mathcal{L} V_{2}(t)\right\} \leq \mathbb{E}\left\{-2 k_{y}\left(\sum_{j=1}^{m} e_{j}^{2}(t)\right)^{\frac{\alpha+1}{2}}\right\}=-2 k_{y} \mathbb{E}\left\{V_{2}^{\frac{\alpha+1}{2}}(t)\right\}
$$

Therefore, according to Definitions 3 and 4, the finite-time antisynchronization for system (5) can be achieved via the controller (9) with $0<\alpha<1$, and the finite times are given as:

$$
\begin{aligned}
& T_{x}=\frac{V^{\frac{1-\alpha}{2}}(0)}{k_{i}(1-\alpha)}=\frac{\left\|e_{i}(0)\right\|^{1-\alpha}}{k_{i}(1-\alpha)}, \\
& T_{y}=\frac{V^{\frac{1-\alpha}{2}}(0)}{k_{j}(1-\alpha)}=\frac{\left\|e_{j}(0)\right\|^{1-\alpha}}{k_{j}(1-\alpha)} .
\end{aligned}
$$

The proof is completed.

Remark 5. The systems (3) and (4) contain the case of $\delta(t) \equiv 1$, $\left(\delta_{0}=1\right), \delta(t) \equiv 0,\left(\delta_{0}=0\right)$ and $\rho(t) \equiv 1,\left(\rho_{0}=1\right), \rho(t) \equiv 0,\left(\rho_{0}=\right.$ 0 ). Thus, some previous study on BAMNNs can be treated as a special case of this paper.

Corollary 1. Suppose Assumption 3 is satisfied, then the error systems (13) and (14) without leakage delay are exponentially stable in the mean square via the controller (9), if there exists the gain constants $\eta_{i}, \eta_{j}$ and $k_{i}, k_{j}$ such that

$$
\left\{\begin{array}{l}
\sum_{i=1}^{n}\left[\left|a_{i}\right|-\sum_{j=1}^{m}\left|\bar{b}_{j i}\right| \bar{L}_{x}-\sum_{j=1}^{m}\left|\bar{c}_{j i}\right| \bar{L}_{x}-R_{1}-R_{3}+2 \eta_{x}\right] \\
-\sum_{i=1}^{m} \sum_{j=1}^{n}\left|\bar{b}_{i j}\right| \bar{L}_{x} \geq 0, \\
\left|\bar{c}_{j i}\right| \bar{L}_{x} \delta_{0}+\frac{1}{m} R_{2} \leq 0, \\
\left|\bar{c}_{j i}\right| \bar{L}_{x}\left(1-\delta_{0}\right)+\frac{1}{m} R_{4} \leq 0,
\end{array}\right.
$$


and

$\left\{\begin{array}{l}\sum_{j=1}^{m}\left[\left|d_{j}\right|-\sum_{i=1}^{n}\left|\bar{q}_{i j}\right| \bar{L}_{y}-\sum_{i=1}^{n}\left|\bar{p}_{i j}\right| \bar{L}_{y}-P_{1}-P_{3}+2 \eta_{y}\right] \\ -\sum_{i=1}^{m} \sum_{j=1}^{n}\left|\bar{q}_{j i}\right| \bar{L}_{y} \geq 0 . \\ \left|\bar{p}_{i j}\right| \bar{L}_{y} \rho_{0}+\frac{1}{n} P_{2} \leq 0 \\ \left|\bar{p}_{i j}\right| \bar{L}_{y}\left(1-\rho_{0}\right)+\frac{1}{n} P_{4} \leq 0 .\end{array}\right.$

where $R_{1}, R_{2}, R_{3}, R_{4}$ and $P_{1}, P_{2}, P_{3}, P_{4}$ are all positive constants determined in the later. Then, the synchronization times are estimated by

$T_{x}=\frac{V^{\frac{1-\alpha}{2}}(0)}{k_{x}(1-\alpha)}=\frac{\left\|e_{i}(0)\right\|^{1-\alpha}}{k_{x}(1-\alpha)}$,

$T_{y}=\frac{V^{\frac{1-\alpha}{2}}(0)}{k_{y}(1-\alpha)}=\frac{\left\|e_{j}(0)\right\|^{1-\alpha}}{k_{y}(1-\alpha)}$

Proof. Let the leakage time delay $\tau=0$ and $\sigma=0$ in Theorem 1 . The proof can be followed, thus it is omitted here.

Remark 6. At present, the research on finite-time stability of the MBAMNNs with time-varying delays and stochastic perturbations is very few. There are numerous models about stability of MBAMNNs within finite-time are special cases of our proposed model. Here we give Theorem 2 about the special case.

Throughout this paper, we consider system (4) without stochastic perturbations as the drive system and corresponding response system is as follows

$$
\begin{aligned}
d \hat{x}_{i}(t) \in & {\left[-a_{i} \hat{x}_{i}(t-\tau)+\sum_{j=1}^{m} \operatorname{co}\left[b_{j i}\left(\hat{x}_{i}(t)\right)\right] f_{j}\left(\hat{y}_{j}(t)\right)\right.} \\
& +\delta(t) \sum_{j=1}^{m} \operatorname{co}\left[c_{j i}\left(\hat{x}_{i}(t-\tau(t))\right)\right] f_{j}\left(\hat{y}_{j}\left(t-\tau_{1}(t)\right)\right) \\
& \left.+(1-\delta(t)) \sum_{j=1}^{m} \operatorname{co}\left[c_{j i}\left(\hat{x}_{i}(t-\tau(t))\right)\right] f_{j}\left(\hat{y}_{j}\left(t-\tau_{2}(t)\right)\right)+u_{i}(t)\right] d t \\
d \hat{y}_{j}(t) \in & {\left[-d_{j} \hat{y}_{j}(t-\sigma)+\sum_{i=1}^{n} \operatorname{co}\left[q_{i j}\left(\hat{y}_{j}(t)\right)\right] g_{i}\left(\hat{x}_{i}(t)\right)\right.} \\
& +\rho(t) \sum_{i=1}^{n} \operatorname{co}\left[p_{i j}\left(\hat{y}_{j}(t-\sigma(t))\right)\right] g_{i}\left(\hat{x}_{i}\left(t-\sigma_{1}(t)\right)\right) \\
& \left.+(1-\rho(t)) \sum_{i=1}^{n} \operatorname{co}\left[p_{i j}\left(\hat{y}_{j}(t-\sigma(t))\right)\right] g_{i}\left(\hat{x}_{i}\left(t-\sigma_{2}(t)\right)\right)+u_{j}(t)\right] d t .
\end{aligned}
$$

Under this case, the error systems (13) and (14) reduce to

$$
\begin{aligned}
d e_{i}(t) \leq & {\left[-a_{i} e_{i}(t-\tau)+\sum_{j=1}^{m} \bar{b}_{j i} F_{j}\left(e_{j}(t)\right)\right.} \\
& +\delta(t) \sum_{j=1}^{m} \bar{c}_{j i} F_{j}\left(e_{j}\left(t-\tau_{1}(t)\right)\right) \\
& \left.+(1-\delta(t)) \sum_{j=1}^{m} \bar{c}_{j i} F_{j}\left(e_{j}\left(t-\tau_{2}(t)\right)\right)+u_{i}(t)\right] d t .
\end{aligned}
$$

and

$$
\begin{aligned}
d e_{j}(t) \leq & {\left[-d_{j} e_{j}(t-\sigma)+\sum_{i=1}^{n} \bar{q}_{i j} G_{i}\left(e_{i}(t)\right)\right.} \\
& +\rho(t) \sum_{i=1}^{n} \bar{p}_{i j} G_{i}\left(e_{i}\left(t-\sigma_{1}(t)\right)\right) \\
& \left.+(1-\rho(t)) \sum_{i=1}^{n} \bar{p}_{i j} G_{i}\left(e_{i}\left(t-\sigma_{2}(t)\right)\right)+u_{j}(t)\right] d t .
\end{aligned}
$$

Thus, we have the following theorem.
Theorem 2. Under Assumptions 1-3, the error systems (29) and (30) of the MBAMNNs with probabilistic time-varying will be globally stable in finite time via the controller (9), if there exists the gain constants $\eta_{x}, \eta_{y}$ and $k_{x}, k_{y}$ such that

$$
\left\{\begin{array}{l}
\sum_{i=1}^{n}\left[\left|a_{i}\right|-\sum_{j=1}^{m}\left|\bar{b}_{j i}\right| \bar{L}_{x}-\sum_{j=1}^{m}\left|\bar{c}_{j i}\right| \bar{L}_{x}+2 \eta_{x}\right] \\
-\sum_{i=1}^{m} \sum_{j=1}^{n}\left|\bar{b}_{i j}\right| \bar{L}_{x} \geq 0 \\
\left|\bar{c}_{j i}\right| \bar{L}_{x} \delta_{0} \leq 0, \quad\left|\bar{c}_{j i}\right| \bar{L}_{x}\left(1-\delta_{0}\right) \leq 0
\end{array}\right.
$$

and

$\left\{\begin{array}{l}\sum_{j=1}^{n}\left[\left|d_{j}\right|-\sum_{i=1}^{n}\left|\bar{q}_{i j}\right| \bar{L}_{y}-\sum_{i=1}^{n}\left|\bar{p}_{i j}\right| L_{y}+2 \eta_{y}\right] \\ -\sum_{i=1}^{m} \sum_{j=1}^{n}\left|\bar{q}_{j i}\right| \bar{L}_{y} \geq 0 . \\ \left|\bar{p}_{i j}\right| \bar{L}_{y} \rho_{0} \leq 0, \quad\left|\bar{p}_{i j}\right| \bar{L}_{y}\left(1-\rho_{0}\right) \leq 0 .\end{array}\right.$

Then, the synchronization time is estimated by

$T_{x}=\frac{V^{\frac{1-\alpha}{2}}(0)}{k_{x}(1-\alpha)}=\frac{\left\|e_{l}(0)\right\|^{1-\alpha}}{k_{x}(1-\alpha)}$,

$T_{y}=\frac{V^{\frac{1-\alpha}{2}}(0)}{k_{y}(1-\alpha)}=\frac{\left\|e_{j}(0)\right\|^{1-\alpha}}{k_{y}(1-\alpha)}$.

Proof. Construct the following Lyapunov function

$V\left(t, e_{i}(t), e_{j}(t)\right)=\sum_{i=1}^{n} e_{i}^{2}(t)+\sum_{j=1}^{m} e_{j}^{2}(t)$

Let

$V_{1}(t)=\sum_{i=1}^{n} e_{i}^{2}(t), V_{2}(t)=\sum_{j=1}^{m} e_{j}^{2}(t)$.

Then $V\left(t, e_{i}(t), e_{j}(t)\right)=V_{1}(t)+V_{2}(t)$.

$\mathbb{E}\left\{\mathcal{L} V\left(t, e_{i}(t), e_{j}(t)\right)\right\}=\mathbb{E}\left\{\mathcal{L} V_{1}(t)\right\}+\mathbb{E}\left\{\mathcal{L} V_{2}(t)\right\}$

By means of Assumptions 2 and 3 and Lemma 1, we get the following inequality And

$$
\begin{aligned}
\mathbb{E}\left\{\mathcal{L} V_{1}(t)\right\}= & 2 \sum_{i=1}^{n} e_{i}(t)\left[-a_{i} e_{i}(t-\tau)+\sum_{j=1}^{m} \bar{b}_{j i} F_{j} e_{j}(t)+u_{i}(t)\right. \\
& +\delta_{0} \sum_{j=1}^{m} \bar{c}_{j i} F_{j} e_{j}\left(t-\tau_{1}(t)\right) \\
& \left.+\left(1-\delta_{0}\right) \sum_{j=1}^{m} \bar{c}_{j i} F_{j} e_{j}\left(t-\tau_{2}(t)\right)\right] .
\end{aligned}
$$

Combine with the Assumption 1 and controller (9), we get

$$
\begin{aligned}
\mathbb{E}\left\{\mathcal{L} V_{1}(t)\right\} & \leq-2 \sum_{i=1}^{n}\left|a_{i}\right| e_{i}(t) e_{i}(t-\tau)+2 \sum_{i=1}^{n} \sum_{j=1}^{m}\left|\bar{b}_{j i}\right| e_{i}(t) F_{j}\left(e_{j}(t)\right) \\
& +2 \sum_{i=1}^{n} \sum_{j=1}^{m} \delta_{0}\left|\bar{c}_{j i}\right| e_{i}(t) F_{j}\left(e_{j}\left(t-\tau_{1}(t)\right)\right) \\
& +2 \sum_{i=1}^{n} \sum_{j=1}^{m}\left(1-\delta_{0}\right)\left|\bar{c}_{j i}\right| e_{i}(t) F_{j}\left(e_{j}\left(t-\tau_{2}(t)\right)\right) \\
& -2 \sum_{i=1}^{n} \eta_{x} e_{i}^{2}(t)-2 \sum_{i=1}^{n} k_{x} e_{i}(t) \operatorname{sign}\left(e_{i}(t)\right)\left|e_{i}(t)\right|^{\alpha},
\end{aligned}
$$


By Eq. (13), we conclude

$$
\begin{aligned}
\mathbb{E}\left\{\mathcal{L} V_{1}(t)\right\} & \leq-\left\{\sum_{i=1}^{n}\left[\left|a_{i}\right|-\sum_{j=1}^{m}\left|\bar{b}_{j i}\right| \bar{L}_{x}-\sum_{j=1}^{m}\left|\bar{c}_{j i}\right| \bar{L}_{x}+2 \eta_{x}\right]\right. \\
& \left.-\sum_{i=1}^{m} \sum_{j=1}^{n}\left|\bar{b}_{i j}\right| \bar{L}_{x}\right\} e_{i}^{2}(t)-\sum_{i=1}^{n}\left|a_{i}\right| e_{i}^{2}(t-\tau) \\
& +\sum_{i=1}^{n}\left[\sum_{j=1}^{m}\left|\bar{c}_{j i}\right| \bar{L}_{x} \delta_{0}\right] e_{j}^{2}\left(t-\tau_{1}(t)\right) \\
& +\sum_{i=1}^{n}\left[\sum_{j=1}^{m}\left|\bar{c}_{j i}\right| \bar{L}_{x}\left(1-\delta_{0}\right)\right] e_{j}^{2}\left(t-\tau_{2}(t)\right) \\
& -2 \sum_{i=1}^{n} k_{x} e_{i}(t) \operatorname{sign}\left(e_{i}(t)\right)\left|e_{i}(t)\right|^{\alpha} .
\end{aligned}
$$

Due to

$\sum_{i=1}^{n} k_{x} e_{i}(t) \operatorname{sign}\left(e_{i}(t)\right)\left|e_{i}(t)\right|^{\alpha}=\sum_{i=1}^{n} k_{x}\left|e_{i}(t)\right|^{\alpha+1}$.

From Lemma 3, We get

$\left(\sum_{i=1}^{n}\left|e_{i}(t)\right|^{\alpha+1}\right)^{\frac{1}{\alpha+1}}=\left(\sum_{i=1}^{n}\left|e_{i}(t)\right|^{2}\right)^{\frac{1}{2}}$.

Hence

$\sum_{i=1}^{n}\left|e_{i}(t)\right|^{\alpha+1}=\left(\sum_{i=1}^{n}\left|e_{i}(t)\right|^{2}\right)^{\frac{\alpha+1}{2}}=\left(\sum_{i=1}^{n} e_{i}^{2}(t)\right)^{\frac{\alpha+1}{2}}$.

Then we conclude

$$
\begin{aligned}
\mathbb{E}\left\{\mathcal{L} V_{1}(t)\right\} & \leq-\left\{\sum_{i=1}^{n}\left[\left|a_{i}\right|-\sum_{j=1}^{m}\left|\bar{b}_{j i}\right| \bar{L}_{x}-\sum_{j=1}^{m}\left|\bar{c}_{j i}\right| \bar{L}_{x}+2 \eta_{x}\right]\right. \\
& \left.-\sum_{i=1}^{m} \sum_{j=1}^{n}\left|\bar{b}_{i j}\right| \bar{L}_{x}\right\} e_{i}^{2}(t)-\sum_{i=1}^{n}\left|a_{i}\right| e_{i}^{2}(t-\tau) \\
& +\sum_{i=1}^{n}\left[\sum_{j=1}^{n}\left|\bar{c}_{j i}\right| \bar{L}_{x} \delta_{0}\right] e_{j}^{2}\left(t-\tau_{1}(t)\right)-2 k_{x}\left(\sum_{i=1}^{n} e_{i}^{2}(t)\right)^{\frac{\alpha+1}{2}} \\
& +\sum_{i=1}^{n}\left[\sum_{j=1}^{n}\left|\bar{c}_{j i}\right| \bar{L}_{x}\left(1-\delta_{0}\right)\right] e_{j}^{2}\left(t-\tau_{2}(t)\right) .
\end{aligned}
$$

So according to Theorem 1 and Lemma 3, we obtain

$$
\mathbb{E}\left\{\mathcal{L} V_{1}(t)\right\} \leq \mathbb{E}\left\{-2 k_{x}\left(\sum_{i=1}^{n} e_{i}^{2}(t)\right)^{\frac{\alpha+1}{2}}\right\}=-2 k_{x} \mathbb{E}\left\{V_{1}^{\frac{\alpha+1}{2}}(t)\right\} .
$$

With the similar process of $\mathcal{L} V_{1}(t)$, we can have

$$
\mathbb{E}\left\{\mathcal{L} V_{2}(t)\right\} \leq \mathbb{E}\left\{-2 k_{y}\left(\sum_{j=1}^{m} e_{j}^{2}(t)\right)^{\frac{\alpha+1}{2}}\right\}=-2 k_{y} \mathbb{E}\left\{V_{2}^{\frac{\alpha+1}{2}}(t)\right\} .
$$

Therefore, according to Definitions 3 and 4, the finite-time antisynchronization for the systems (3) and (28) can be achieved via the controller (9) with $0<\alpha<1$, and the finite times are given as:

$T_{x}=\frac{V^{\frac{1-\alpha}{2}}(0)}{k_{x}(1-\alpha)}=\frac{\left\|e_{i}(0)\right\|^{1-\alpha}}{k_{x}(1-\alpha)}$.

$T_{y}=\frac{V^{\frac{1-\alpha}{2}}(0)}{k_{y}(1-\alpha)}=\frac{\left\|e_{j}(0)\right\|^{1-\alpha}}{k_{y}(1-\alpha)}$.

The proof is completed.

Corollary 2. Suppose Assumption 3 is satisfied, then the error systems (29) and (30) without leakage delay are globally exponentially stable in the mean square via the controllers (9), if there exists the gain constants $\eta_{x}, \eta_{y}$ and $k_{x}, k_{y}$ such that

$$
\left\{\begin{array}{l}
\sum_{i=1}^{n}\left[\left|a_{i}\right|-\sum_{j=1}^{m}\left|\bar{b}_{j i}\right| \bar{L}_{x}-\sum_{j=1}^{m}\left|\bar{c}_{j i}\right| \bar{L}_{x}+2 \eta_{x}\right] \\
-\sum_{i=1}^{m} \sum_{j=1}^{n}\left|\bar{b}_{i j}\right| \bar{L}_{x} \geq 0, \\
\left|\bar{c}_{j i}\right| \bar{L}_{x} \delta_{0} \leq 0, \quad\left|\bar{c}_{j i}\right| \bar{L}_{x}\left(1-\delta_{0}\right) \leq 0,
\end{array}\right.
$$

and

$$
\left\{\begin{array}{l}
\sum_{j=1}^{m}\left[\left|d_{j}\right|-\sum_{i=1}^{n}\left|\bar{q}_{i j}\right| \bar{L}_{y}-\sum_{i=1}^{n}\left|\bar{p}_{i j}\right| \bar{L}_{y}+2 \eta_{y}\right] \\
-\sum_{i=1}^{m} \sum_{j=1}^{n}\left|\bar{q}_{j i}\right| \bar{L}_{y} \geq 0 \\
\left|\bar{p}_{i j}\right| \bar{L}_{y} \rho_{0} \leq 0, \quad\left|\bar{p}_{i j}\right| \bar{L}_{y}\left(1-\rho_{0}\right) \leq 0 .
\end{array}\right.
$$

Then, the synchronization time is estimated by

$$
\begin{aligned}
& T_{x}=\frac{V^{\frac{1-\alpha}{2}}(0)}{k_{x}(1-\alpha)}=\frac{\left\|e_{i}(0)\right\|^{1-\alpha}}{k_{x}(1-\alpha)}, \\
& T_{y}=\frac{V^{\frac{1-\alpha}{2}}(0)}{k_{y}(1-\alpha)}=\frac{\left\|e_{j}(0)\right\|^{1-\alpha}}{k_{y}(1-\alpha)} .
\end{aligned}
$$

Proof. Let the leakage time delay $\tau=0$ and $\sigma=0$ in Theorem 2. The proof is omitted here.

Remark 7. Due to the condition of the time-varying delays $\tau(t)$, $\sigma(t)$, Theorem 2 provides a special case of Theorem 1. It should be mentioned that no complex numerical calculation such as computing redundance algebraic criterions [58] or solving linear matrix inequality (LMIs) $[59,60]$ is needed in the anti-synchronization conditions. Thus, our anti-synchronization consequences have a wider adaptive capability and more successful application.

Remark 8. There is no extra demand on activation functions but requesting they are bounded and the time-varying delays are probabilistic. Furthermore, overall consideration of our obtained results with finite time anti-synchronization, it can be expected to have a powerful potential application in areas such as secret communication, image encryption, digital processing, and so on.

\section{Numerical simulation}

To show the effectiveness of the obtained results, several numerical simulations are presented as follows. There exist $b_{j i}(t) \in \operatorname{co}\left[b_{j i}\left(x_{i}(t)\right)\right], c_{j i}(t) \in \operatorname{co}\left[c_{j i}\left(x_{i}(t-\tau(t))\right)\right], \quad q_{i j}(t) \in \operatorname{co}\left[q_{i j}\left(y_{j}(t)\right)\right]$, $p_{i j}(t) \in \operatorname{co}\left[p_{i j}\left(y_{j}(t-\sigma(t))\right)\right]$, so the drive system be considered as follows

$$
\begin{aligned}
\dot{x}_{i}(t)= & -a_{i} x_{i}(t-\tau)+\sum_{j=1}^{2} b_{j i}(t) f_{j}\left(y_{j}(t)\right) \\
& +\delta_{0} \sum_{j=1}^{2} c_{j i}(t) g_{j}\left(y_{j}\left(t-\tau_{1}(t)\right)\right) \\
& +\left(1-\delta_{0}\right) \sum_{j=1}^{2} c_{j i}(t) g_{j}\left(y_{j}\left(t-\tau_{2}(t)\right)\right), \\
\dot{y}_{j}(t)= & -d_{j} y_{j}(t-\sigma)+\sum_{i=1}^{2} q_{i j}(t) f_{i}\left(x_{i}(t)\right) \\
& +\rho_{0} \sum_{i=1}^{2} p_{i j}(t) g_{i}\left(x_{i}\left(t-\sigma_{1}(t)\right)\right) \\
& +\left(1-\rho_{0}\right) \sum_{i=1}^{2} p_{i j}(t) g_{i}\left(x_{i}\left(t-\sigma_{2}(t)\right)\right) .
\end{aligned}
$$


And the response system is defined as

$$
\begin{aligned}
d \hat{x}_{i}(t)= & {\left[-a_{i} \hat{x}_{i}(t-\tau)+\sum_{j=1}^{2} b_{j i}(t) f_{j}\left(\hat{y}_{j}(t)\right)\right.} \\
& +\delta_{0} \sum_{j=1}^{2} c_{j i}(t) g_{j}\left(\hat{y}_{j}\left(t-\tau_{1}(t)\right)\right) \\
& \left.+\left(1-\delta_{0}\right) \sum_{j=1}^{2} c_{j i}(t) g_{j}\left(\hat{y}_{j}\left(t-\tau_{2}(t)\right)\right)+u_{i}(t)\right] d t \\
& +\sum_{j=1}^{2} \beta_{j i}\left(t . e_{j}(t), e_{j}\left(t-\tau_{1}(t)\right)\right) d \omega_{j}(t) \\
& +\sum_{j=1}^{2} \beta_{j i}\left(t, e_{j}(t), e_{j}\left(t-\tau_{2}(t)\right)\right) d \omega_{j}(t),
\end{aligned}
$$

$$
\begin{aligned}
d \hat{y}_{j}(t)= & {\left[-d_{j} \hat{y}_{j}(t-\sigma)+\sum_{i=1}^{2} q_{i j}(t) f_{i}\left(\hat{x}_{i}(t)\right)\right.} \\
& +\rho_{0} \sum_{i=1}^{2} p_{i j}(t) g_{i}\left(\hat{x}_{i}\left(t-\sigma_{1}(t)\right)\right) \\
& \left.+\left(1-\rho_{0}\right) \sum_{i=1}^{2} p_{i j}(t) g_{i}\left(\hat{x}_{i}\left(t-\sigma_{2}(t)\right)\right)+u_{j}(t)\right] d t \\
& +\sum_{i=1}^{2} \beta_{i j}\left(t, e_{i}(t), e_{i}\left(t-\sigma_{1}(t)\right)\right) d \omega_{i}(t) \\
& +\sum_{i=1}^{2} \beta_{i j}\left(t, e_{i}(t) \cdot e_{i}\left(t-\sigma_{2}(t)\right)\right) d \omega_{i}(t) .
\end{aligned}
$$

Taking the activation function as $g(\cdot)=\tanh (|\cdot|-1)$ and $\quad f()=.\sin (|\cdot|-1) . \quad \tau_{1}(t)=\sigma_{1}(t)=0.75-0.25 \cos (t)$, $\tau_{2}(t)=\sigma_{2}(t)=0.75-0.25 \sin (t)$. We have $\tau=\sigma=0.15$ $\delta_{0}=\rho_{0}=0.2$.

$$
\mathbf{a}=\left[\begin{array}{ll}
1 & 0 \\
0 & 1
\end{array}\right], \quad \mathbf{d}=\left[\begin{array}{ll}
1 & 0 \\
0 & 1
\end{array}\right] .
$$$$
b_{11}\left(x_{1}(t)\right)= \begin{cases}1.0, & \left|x_{1}(t)\right| \leq 1 \\ 1.0, & \left|x_{1}(t)\right|>1\end{cases}
$$$$
b_{12}\left(x_{1}(t)\right)= \begin{cases}7.0, & \left|x_{1}(t)\right| \leq 1, \\ 5.0, & \left|x_{1}(t)\right|>1,\end{cases}
$$$$
\begin{aligned}
& b_{21}\left(x_{2}(t)\right)= \begin{cases}1.8, & \left|x_{2}(t)\right| \leq 1, \\
1.8, & \left|x_{2}(t)\right|>1,\end{cases} \\
& b_{22}\left(x_{2}(t)\right)= \begin{cases}0.8, & \left|x_{2}(t)\right| \leq 1, \\
1.0, & \left|x_{2}(t)\right|>1,\end{cases}
\end{aligned}
$$$$
q_{11}\left(y_{1}(t)\right)= \begin{cases}1.1, & \left|y_{1}(t)\right| \leq 2, \\ 2.0, & \left|y_{1}(t)\right|>2,\end{cases}
$$$$
q_{12}\left(y_{1}(t)\right)= \begin{cases}6.8, & \left|y_{1}(t)\right| \leq 2, \\ 4.6, & \left|y_{1}(t)\right|>2,\end{cases}
$$

$q_{21}\left(y_{2}(t)\right)= \begin{cases}1.5, & \left|y_{2}(t)\right| \leq 2, \\ 1.5, & \left|y_{2}(t)\right|>2,\end{cases}$

$q_{22}\left(y_{2}(t)\right)= \begin{cases}0.9, & \left|y_{2}(t)\right| \leq 2, \\ 1.2, & \left|y_{2}(t)\right|>2,\end{cases}$

$$
\begin{aligned}
& c_{11}\left(x_{1}(t-\tau(t))\right)= \begin{cases}-1.5, & \left|x_{1}(t-\tau(t))\right| \leq 1, \\
-1.2, & \left|x_{1}(t-\tau(t))\right|>1,\end{cases} \\
& c_{12}\left(x_{1}(t-\tau(t))\right)= \begin{cases}1.0, & \left|x_{1}(t-\tau(t))\right| \leq 1, \\
0.8, & \left|x_{1}(t-\tau(t))\right|>1,\end{cases} \\
& c_{21}\left(x_{2}(t-\tau(t))\right)= \begin{cases}0.8, & \left|x_{2}(t-\tau(t))\right| \leq 1, \\
1.0, & \left|x_{2}(t-\tau(t))\right|>1,\end{cases} \\
& c_{22}\left(x_{2}(t-\tau(t))\right)= \begin{cases}-1.4, & \left|x_{2}(t-\tau(t))\right| \leq 1, \\
-1.6, & \left|x_{2}(t-\tau(t))\right|>1,\end{cases} \\
& p_{11}\left(y_{1}(t-\sigma(t))\right)= \begin{cases}-1.3, & \left|y_{1}(t-\sigma(t))\right| \leq 2, \\
-1.1, & \left|y_{1}(t-\sigma(t))\right|>2,\end{cases} \\
& p_{12}\left(y_{1}(t-\sigma(t))\right)= \begin{cases}0.7, & \left|y_{1}(t-\sigma(t))\right| \leq 2, \\
0.9, & \left|y_{1}(t-\sigma(t))\right|>2,\end{cases} \\
& p_{21}\left(y_{2}(t-\sigma(t))\right)= \begin{cases}0.9, & \left|y_{2}(t-\sigma(t))\right| \leq 2, \\
1.2, & \left|y_{2}(t-\sigma(t))\right|>2,\end{cases} \\
& p_{22}\left(y_{2}(t-\sigma(t))\right)= \begin{cases}-1.3, & \left|y_{2}(t-\sigma(t))\right| \leq 2, \\
-1.7, & \left|y_{2}(t-\sigma(t))\right|>2 .\end{cases}
\end{aligned}
$$

For the controllers, we let $k_{x}=\operatorname{diag}\{1,1\}, \quad k_{y}=\operatorname{diag}\{1,1\}$ and $\quad \eta_{x}=\operatorname{diag}\{115.160\}, \quad \eta_{y}=\operatorname{diag}\{175,177\} \quad$ We choose $\alpha=0.6$, and the initial values of the state variables as $\quad\left[x_{1}(t), x_{2}(t)\right]=[-1.45 .1 .6] . \quad\left[\hat{x}_{1}(t), \hat{x}_{2}(t)\right]=[-1.35,1.5]$, $\left[y_{1}(t), y_{2}(t)\right]=[-1.95,1.2]$ and $\left[\hat{y}_{1}(t), \hat{y}_{2}(t)\right]=[-1.85,1.3]$.

The Brownian motion satisfies $E \omega(t)=0, D \omega(t)=1$.

$$
\begin{aligned}
& \beta_{\mathbf{j i}}\left(\mathbf{t}, \mathbf{e}_{\mathbf{j}}(\mathbf{t}), \mathbf{e}_{\mathbf{j}}\left(\mathbf{t}-\tau_{\mathbf{1}}(\mathbf{t})\right)=\operatorname{diag}\left\{0.4 e_{1}(t)+0.3 e_{1}\left(t-\tau_{1}(t)\right),\right.\right. \\
& \left.\quad-0.5 e_{2}(t)+0.2 e_{2}\left(t-\tau_{1}(t)\right)\right\}, \\
& \beta_{\mathbf{j i}}\left(\mathbf{t} . \mathbf{e}_{\mathbf{j}}(\mathbf{t}), \mathbf{e}_{\mathbf{j}}\left(\mathbf{t}-\tau_{\mathbf{2}}(\mathbf{t})\right)=\operatorname{diag}\left\{0.4 e_{1}(t)+0.4 e_{1}\left(t-\tau_{2}(t)\right),\right.\right. \\
& \left.\quad-0.5 e_{2}(t)+0.2 e_{2}\left(t-\tau_{2}(t)\right)\right\}, \\
& \beta_{\mathbf{i j}}\left(\mathbf{t}, \mathbf{e}_{\mathbf{i}}(\mathbf{t}), \mathbf{e}_{\mathbf{i}}\left(\mathbf{t}-\sigma_{1}(\mathbf{t})\right)=\operatorname{diag}\left\{0.4 e_{1}(t)+0.3 e_{1}\left(t-\sigma_{1}(t)\right),\right.\right. \\
& \left.\quad-0.5 e_{2}(t)+0.2 e_{2}\left(t-\sigma_{1}(t)\right)\right\}, \\
& \beta_{\mathbf{i j}}\left(\mathbf{t} . \mathbf{e}_{\mathbf{i}}(\mathbf{t}), \mathbf{e}_{\mathbf{i}}\left(\mathbf{t}-\sigma_{\mathbf{2}}(\mathbf{t})\right)=\operatorname{diag}\left\{0.4 e_{1}(t)+0.3 e_{1}\left(t-\sigma_{2}(t)\right),\right.\right. \\
& \left.\quad-0.5 e_{2}(t)+0.2 e_{2}\left(t-\sigma_{2}(t)\right)\right\} .
\end{aligned}
$$

Fig. 2 shows that the drive system (3) has a limit cycle in the case of the above-mentioned parameters. It is clear that drive system (3) and the corresponding response system (4) are globally anti-synchronized in the mean square. Figs. 3 and 4 depict the state trajectories and anti-synchronization error of the state variables between systems (3) and (4), respectively.

Get together the above mentioned parameters with the condition (27), we calculate the setting times $T_{x 1}=T_{x 2}=T_{y 1}=T_{y 2}=$ 0.995 . It is easy to see that the states are quickly converging to stable according to Fig. 4 within the setting time. Hence, it can be concluded that, according to Theorem 1, the considered system (3) can be globally anti-synchronized with system (4) in the mean square.

The drive system (3) without leakage delays has chaotic attractors under the initial values which can be seen in Fig. 5. It follows from Corollary 1, that drive system (3) and the corresponding response system (4) without leakage delays are global antisynchronized in the mean square. Fig. 6 depict the state trajectories and anti-synchronization error of the state variables $x_{1}(t)+$ $\hat{x}_{1}(t), x_{2}(t)+\hat{x}_{2}(t), y_{1}(t)+\hat{y}_{1}(t)$, and $y_{2}(t)+\hat{y}_{2}(t)$ between systems (3) and (4) without leakage delays, respectively. From Fig. 7 , the results shown the feedback control inputs contribute to the chaos anti-synchronization in the mean square.

It is shown that drive system (3) and the corresponding response system (28) are exponentially anti-synchronized in the 


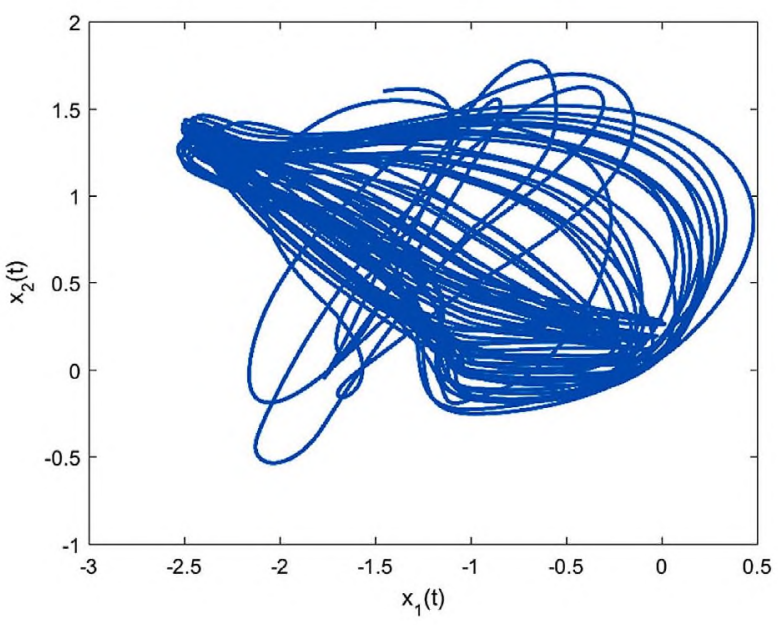

(a)

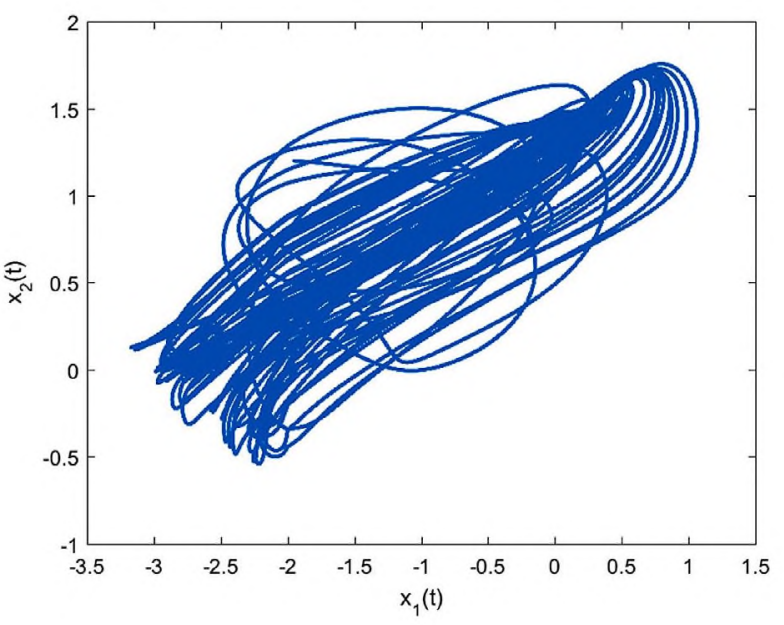

(b)

Fig. 2. Phase trajectories of the drive system (3).

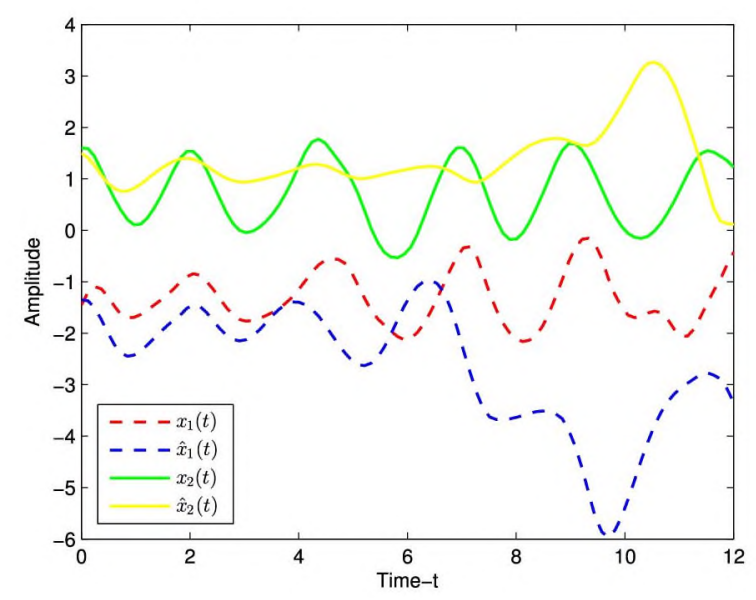

(a)

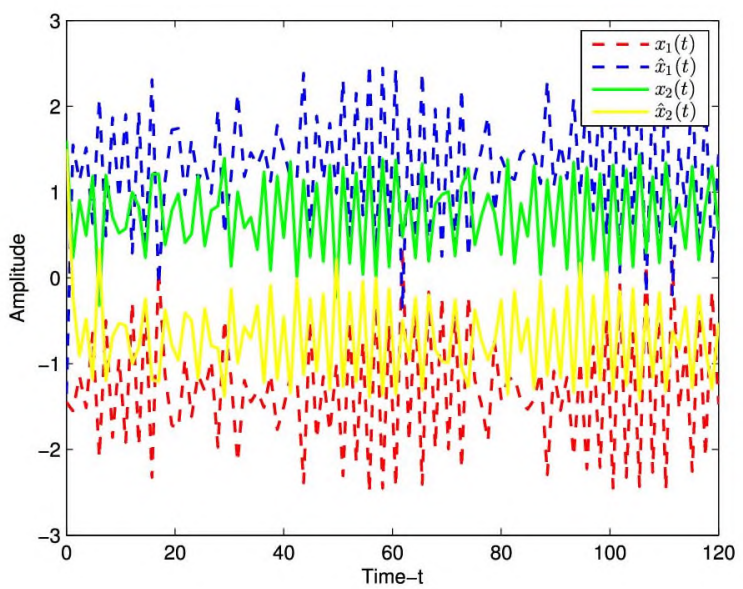

(c)

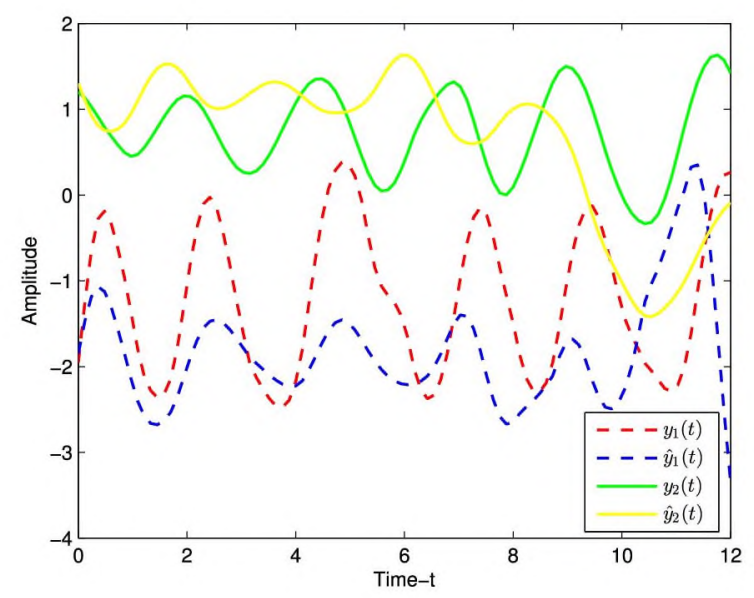

(b)

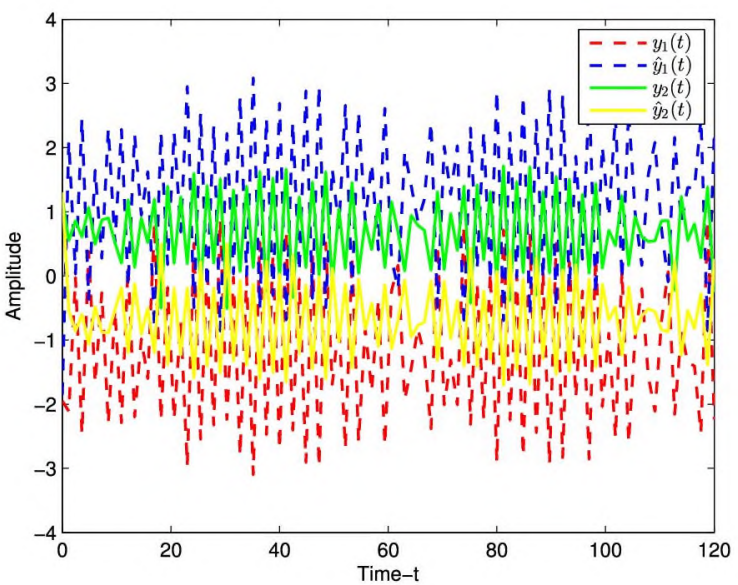

(d)

Fig. 3. (a) and (b) State trajectories of the systems (3) and (4) without control; (c) and (d) State trajectories of the systems (3) and (4) under control, respectively. 


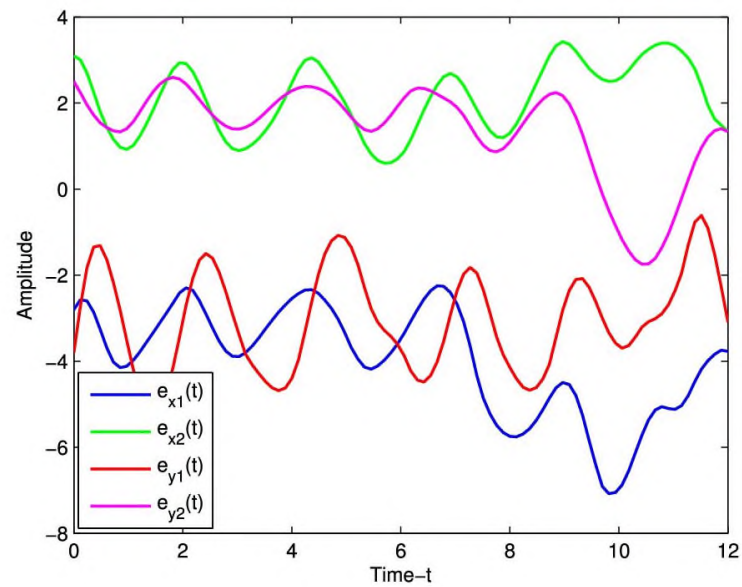

(a)

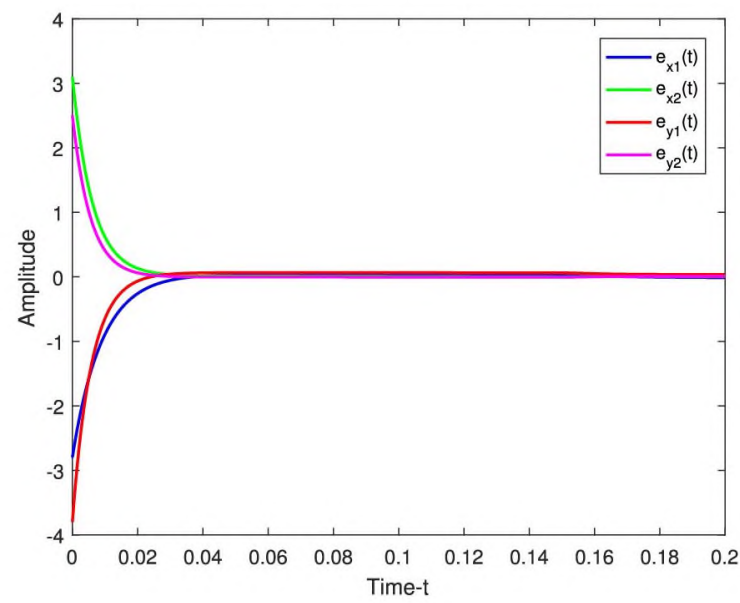

(b)

Fig. 4. (a) The anti-synchronization error of the systems (3) and (4) without control ; (b) The anti-synchronization error of the systems (3) and (4) under control.

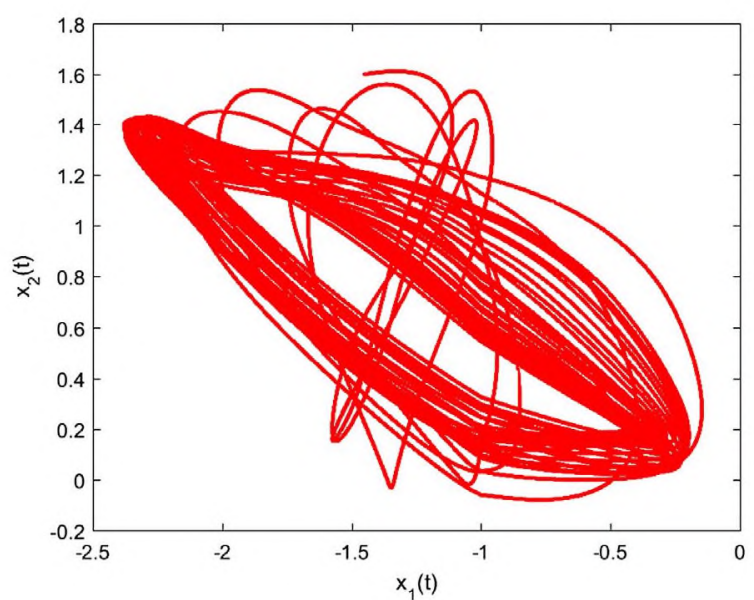

(a)

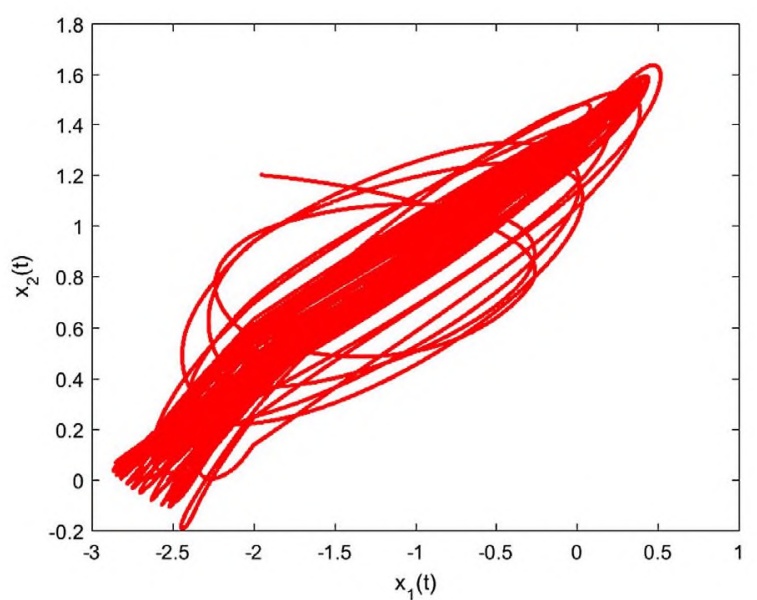

(b)

Fig. 5. Phase trajectories of the drive system (3) without leakage delays.

mean square. Figs. 8 and 9 illustrated the state trajectories and anti-synchronization error of the state variables between systems (3) and (28), respectively. It is easy to see that the states are quickly converging to stable according to Fig. 9. Hence, it can be concluded that, according to Theorem 2, the considered BAM system (3) can be globally anti-synchronized with system (28) in the mean square.

The drive system (3) without leakage delays has chaotic attractors with the initial values which can be seen in Fig. 6. From Figs. 10 to 11, it is observed that the state trajectories of the considered MBAMNNs converge to zero globally in the mean square. Therefore it follows, from Corollary 2, the error systems (29) and (30) are stable in the mean square. This implies that the system (9) is a proper feedback controller to the chaos antisynchronization.

\section{Conclusion}

We have investigated the finite-time anti-synchronization of stochastic MBAMNNs with probabilistic and leakage time-varying delays. The mixed time-varying delays involve discrete time delays, probabilistic time-varying delays and a constant delay in the leakage term. We also considered the stochastic perturbations corresponding to the probabilistic time-varying delays. Based on an effective Lyapunov function, finite-time stability theorem, stochastic analysis theory and Wirtinger-type inequality, sufficient conditions that depend on the time-varying delays are derived to ensure that the MBAMNNs achieve anti-synchronization in the mean square within finite-time. Numerical examples were provided to demonstrate the usefulness and effectiveness of the proposed control strategy. The obtained results extend and improve some previous works on conventional BAMNNs.

The future work mainly includes the following aspects: (1) MBAMNNs is a new and interesting topic, searching for more practical and complex MBAMNNs model is our further work. Since the MBAMNNs can be treated as a discontinuous switched system, it is significant to address a more preferable mathematical method to study: (2) So far, we have completed the application of MNNs to secure communication and image encryption. How to apply the results in practice, such as the associative memory and forget of brain-like, mass storage, machine learning, and so on. In summary, 


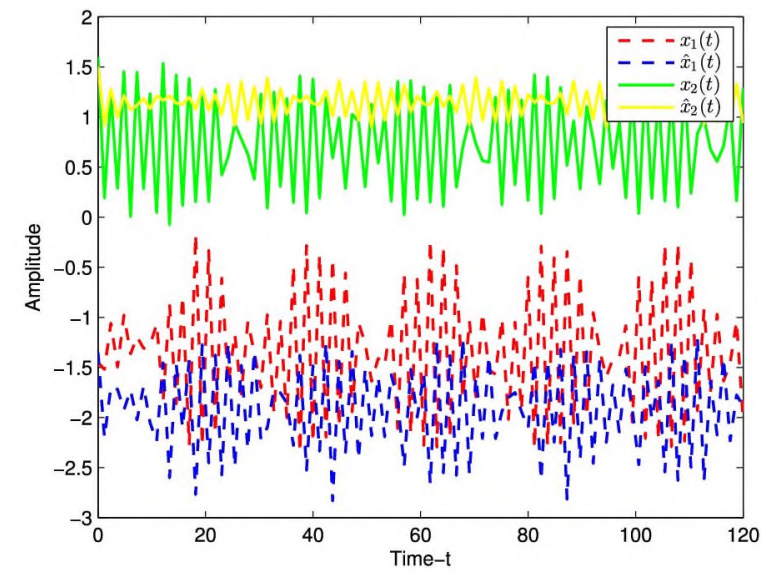

(a)

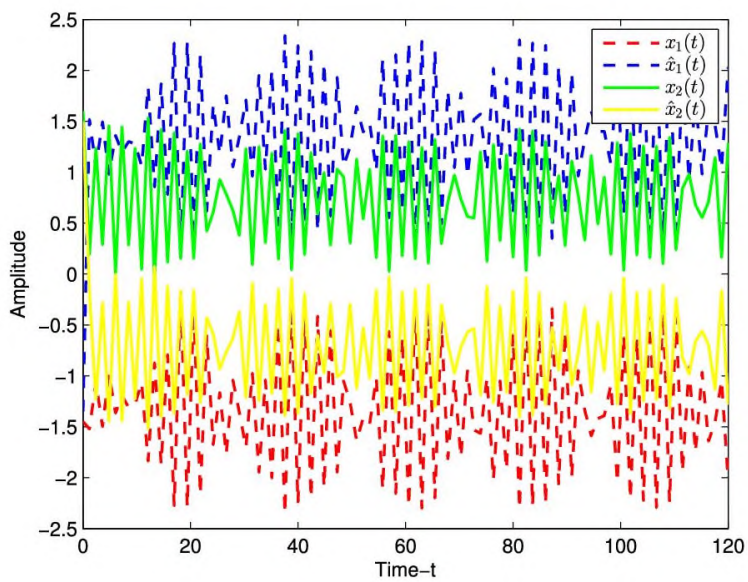

(c)

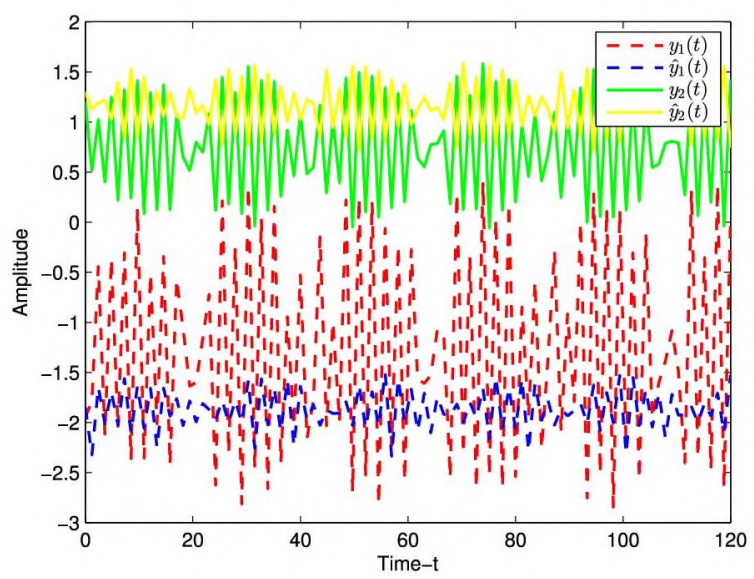

(b)

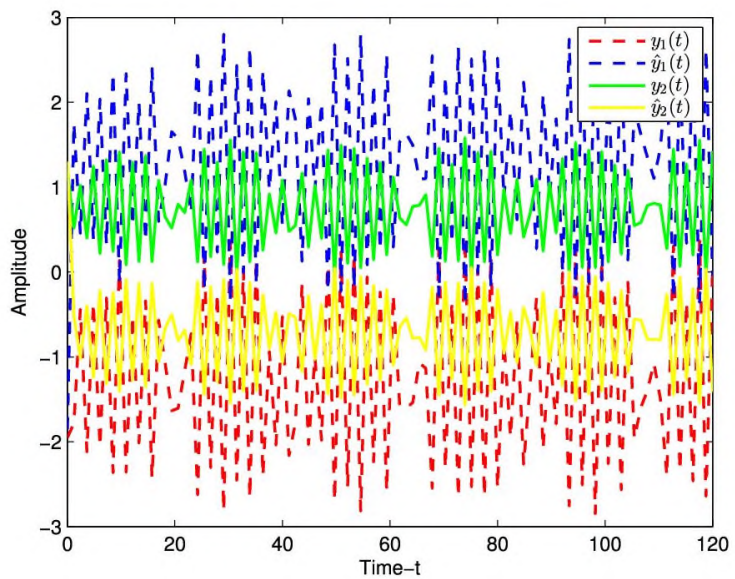

(d)

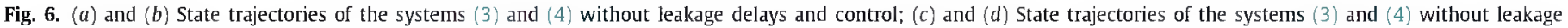
delays but under control, respectively.

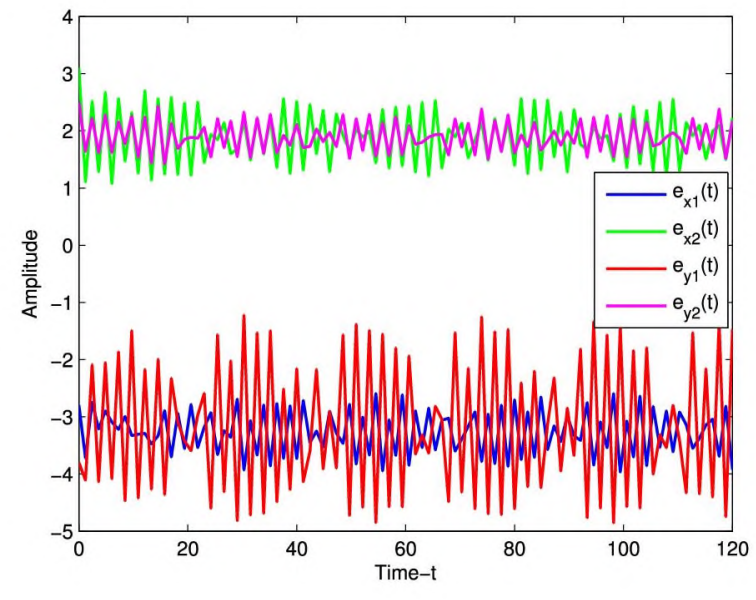

(a)

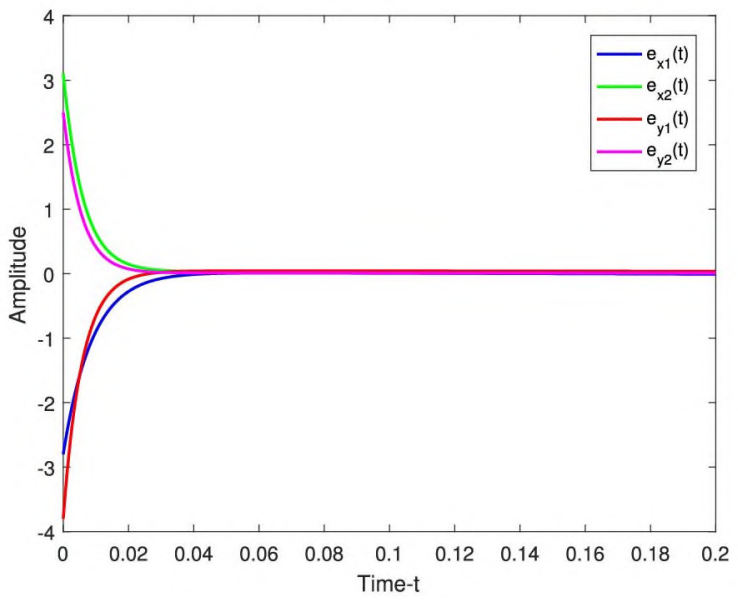

(b)

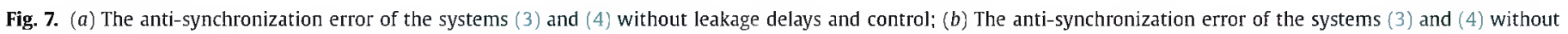
leakage delays but under control. 


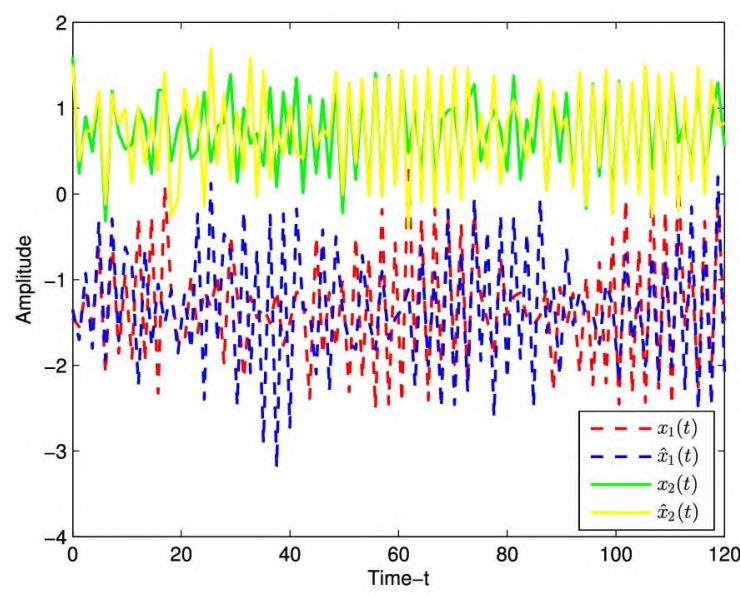

(a)

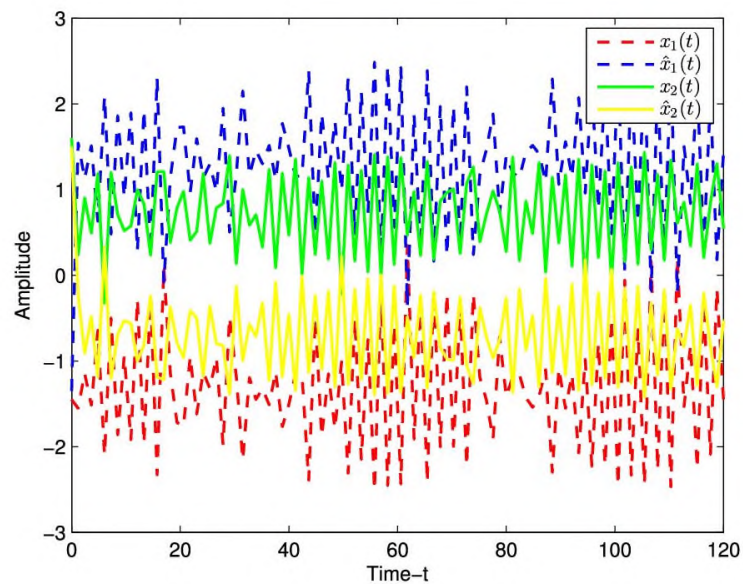

(c)

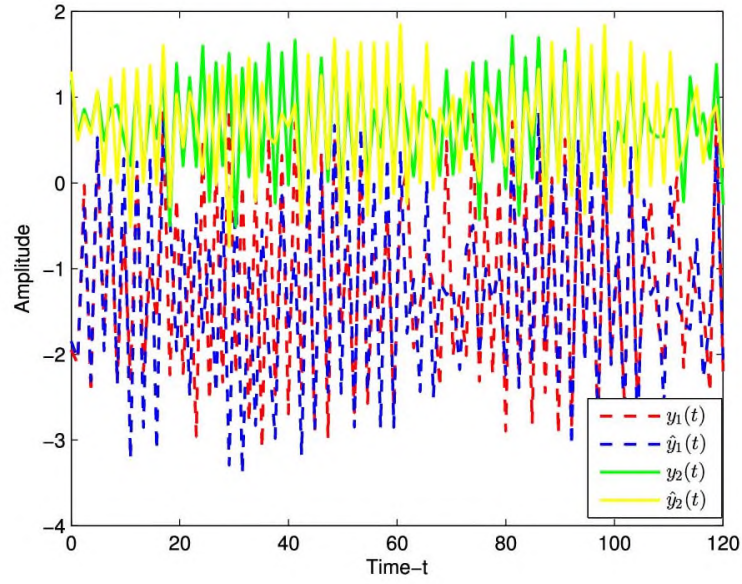

(b)

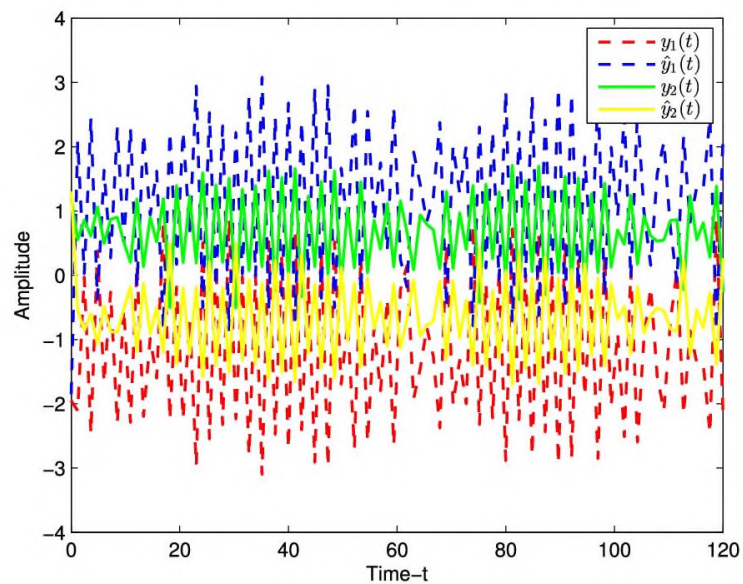

(d)

Fig. 8. (a) and (b) State trajectories of the systems (3) and (28) without control; (c) and (d) State trajectories of the systems (3) and (28) under control, respectively.

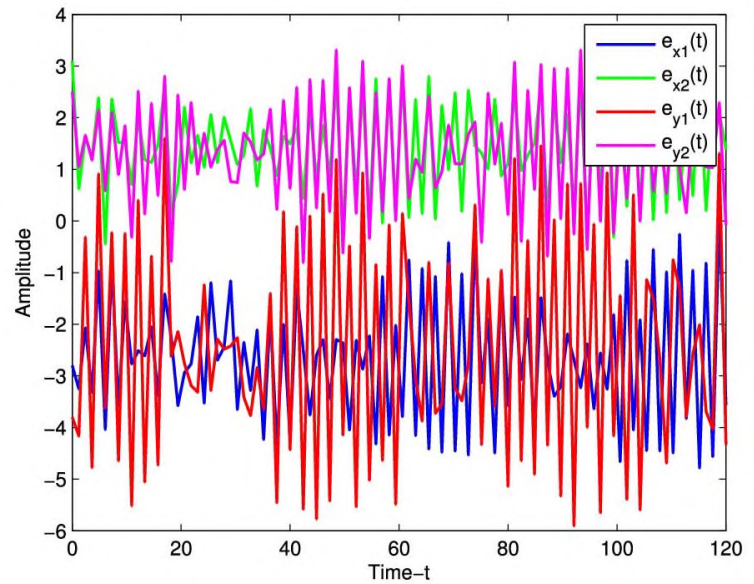

(a)

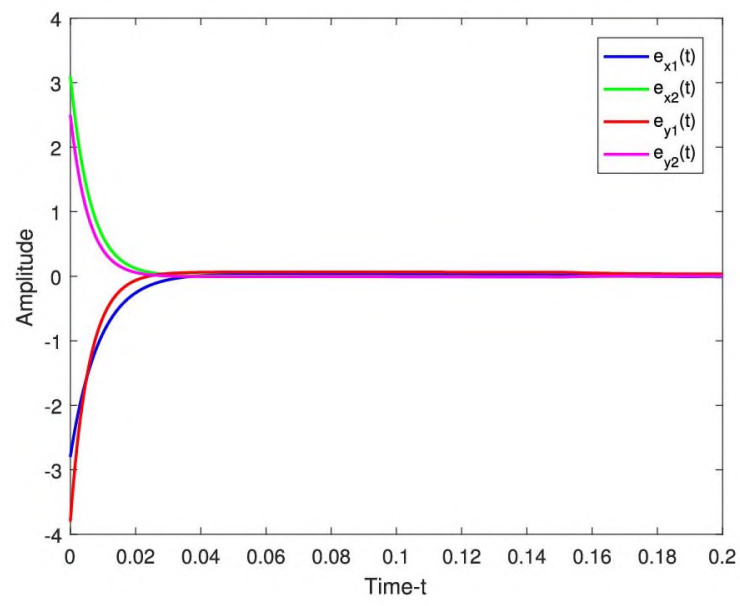

(b)

Fig. 9. (a) The anti-synchronization error of the systems (3) and (28) without control; (b) The anti-synchronization error of the systems (3) and (28) under control. 


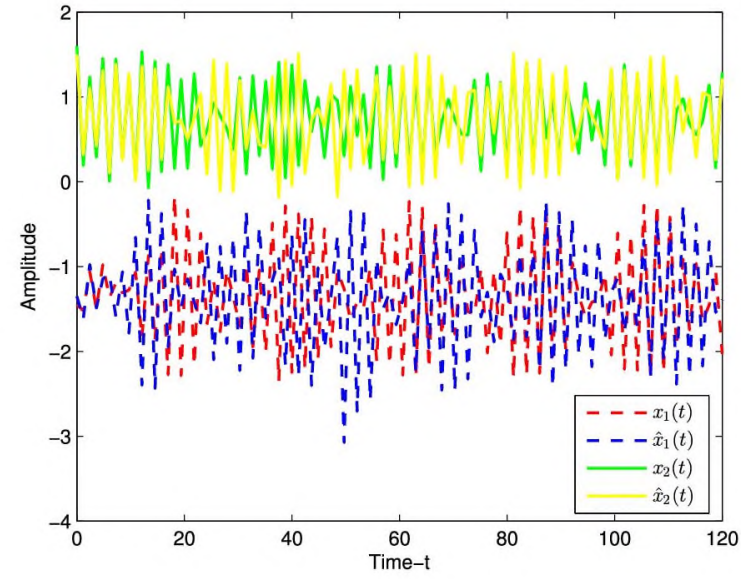

(a)

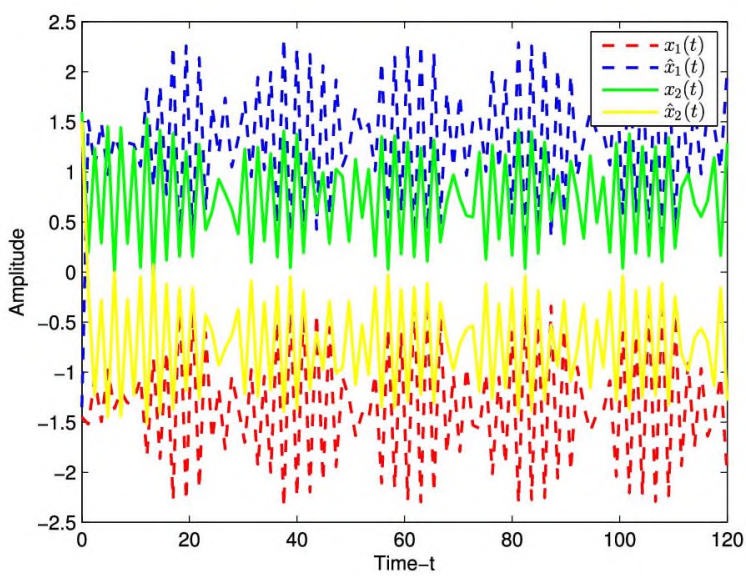

(c)

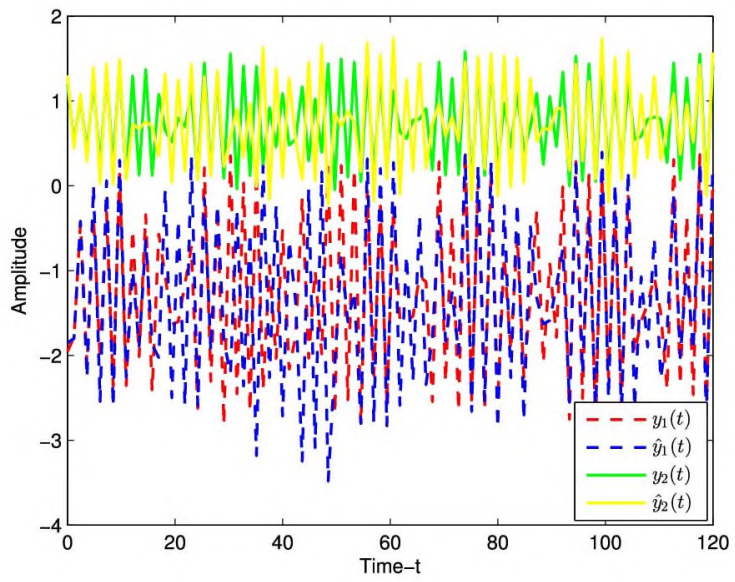

(b)

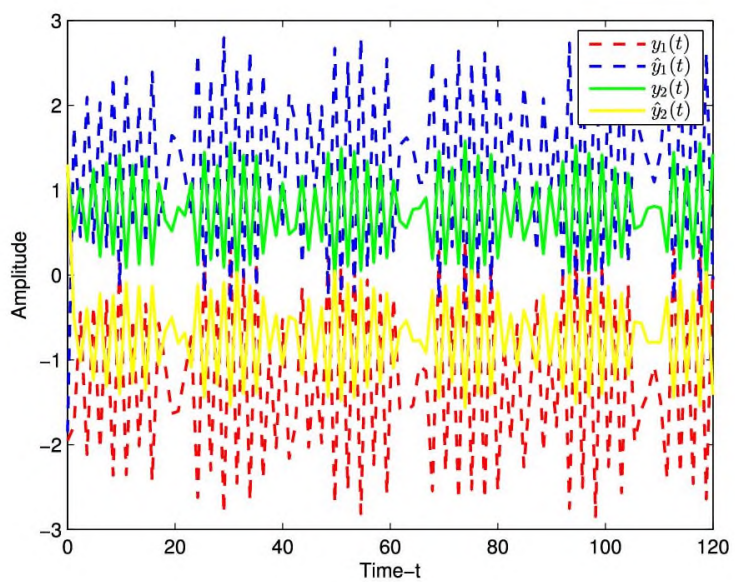

(d)

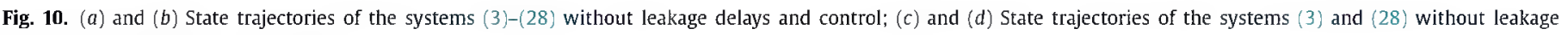
delays but under control, respectively.

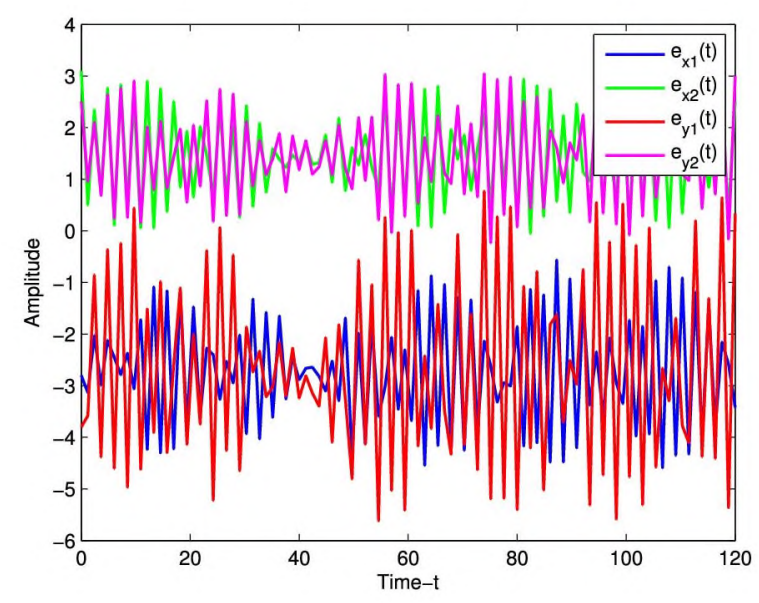

(a)

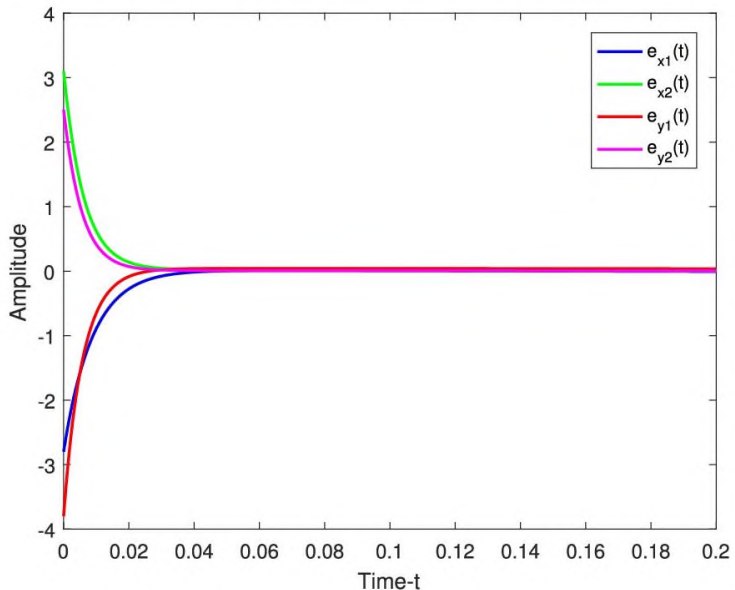

(b)

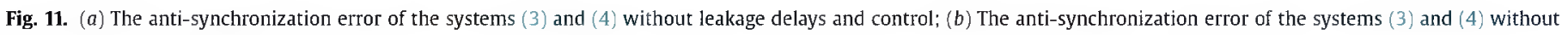
leakage delays but under control. 
the memristive associative memory neural networks still have a lot of problems worthy of further study.

\section{Acknowledgments}

This work was supported by the National Key Research and Development Program of China under Grants 2017YFB0702300, the State Scholarship Fund of China Scholarship Council (CSC), the National Natural Science Foundation of China under Grants 61603032 and 61174103, the Fundamental Research Funds for the Central Universities under Grant 06500025, the National Key Technologies R\&D Program of China under Grant 2015BAK38B01, and the University of Science and Technology Beijing-National Taipei University of Technology Joint Research Program under Grant TW201705.

\section{References}

[1] Sakthivel R, Vadivel P, Mathiyalagan K, Arunkumar A, Sivachitra M. Design of state estimator for bidirectional associative memory neural networks with leakage delays. Inf Sci 2015:296:263-74.

[2] Cao J, Wan Y. Matrix measure strategies for stability and synchronization of inertial BAM neural network with time delays. Neural Netw 2014:53(5):165-72.

[3] Du Y, Zhong S, Zhou N. Nie L, Wang W. Exponential passivity of BAM neural networks with time-varying delays. Appl Math Comput 2013;221:727-40.

[4] Sakthivel R, Arunkumar A, Mathiyalagan K, Anthoni SM. Robust passivity analysis of fuzzy Cohen-Grossberg BAM neural networks with time-varying delays. Appl Math Comput 2011;218:3799-809.

[5] Senan S, Arik S, Liu D. New robust stability results for bidirectional associative memory neural networks with multiple time delays. Appl Math Comput 2012:218:11472-82

[6] Luo $X$, Deng J, Liu J, Wang W, Ban $X_{1}$, Wang J. A quantized kernel least mean square scheme with entropy-guided learning for intelligent data analysis. China Commun 2017:14(7):1-10.

[7] Luo X, Deng J, Wang W, Wang J, Zhao W. A quantized kernel learning algorithm using a minimum kernel risk-sensitive loss criterion and bilateral gradient technique. Entropy 2017;19(7):365

[8] Yu Y, Xiao G, Zhou J, Wang Y, Wang Z, Kurths J, Schellnhuber H. System crash as dynamics of complex networks. Proc Natl Acad Sci USA 2016;113(42):11726.

[9] Wang Z, Bauch C, Bhattacharyya S, et al. Statistical physics of vaccination. Phys Rep 2016:664

[10] Chua L. Memristor-the missing circuit element. IEEE Trans Circuit Theory $1971 ; 18: 507-19$

[11] Gu H. Adaptive synchronization for competitive neural networks with different time scales and stochastic perturbation. Neurocomputing 2009;73:350-6.

[12] Julien B, Gregory S, Philip J, Joshua Y, Duncan R, Stanley W. 'Memristive' switches enable 'stateful' logic operations via material implication. Nature 2010;464:873-6.

[13] Maan A, Jayadevi D, James A. A survey of memristive threshold logic circuits. IEEE Trans Neural Netw Learn Syst 2016:2547842. doi: 10.1109/TNNLS.

[14] Di Ventra M, Pershin Y, Chua L. Circuit elements with memory: memristors, memcapacitors, and meminductors. Proc IEEE 2009;97:1715-16.

[15] Pershin Y, Di Ventra M. Experimental demonstration of associative memory with memristive neural networks. Neural Netw 2010;20:881-6.

[16] Bao H. Park J, Cao J. Exponential synchronization of coupled stochastic memristor-based neural networks with time-varying probabilistic delay coupling and impulsive delay. IEEE Trans Neural Netw Learn Syst 2016;27:190-206.

[17] Tu Z, Ding N, Li L, et al. Adaptive synchronization of memristive neural networks with time-varying delays and reaction-diffusion term. Appl Math Comput 2017;311:118-28.

[18] Wang F, Chen Y, Liu M. pth Moment exponential stability of stochastic memristor-based bidirectional associative memory (BAM) neural networks with time delays. Neura! Netw Off I Int Neural Netw Soc 2017:98:192.

[19] Zheng $\mathrm{M}_{4}$ Li L, Peng $\mathrm{H}_{\text {, et }}$ al. Fixed-time synchronization of memristive fuzzy BAM cellular neural networks with time-varying delays based on feedback controllers. IEEE Access 2018;99:1.

[20] Yuan $M$, Luo $X$, Wang $W$, et al. Pinning synchronization of coupled memristive recurrent neural networks with mixed time-varying delays and perturbations. Neural Process Lett 2018:1-24.

[21] Wang W, Yuan M, Luo X et al. Anti-synchronization control of BAM memristive neural networks with multiple proportional delays and stochastic perturbations. Mod Phys Lett B 2018:3:1850028.

[22] Wang $W$, Wang $M$, Luo $X$, et al. Passivity of memristive BAM neural networks with leakage and additive time-varying delays. Chaos Solitons Fract 2018:1:84-97.

[23] $\mathrm{Hu} B$, Guan $\mathrm{ZH}$, Xiong $N$, et al. Intelligent impulsive synchronization of nonlinear interconnected neural networks for image protection. IEEE Trans Ind Inf 2018;99. 1-1

[24] Wen S, Zeng Z, Huang T, Meng Q, Wei Y. Lag synchronization of switched neural networks via neural activation function and applications in image encryption. IEEE Trans Neural Netw Learn Syst 2015:26:1493-502.
[25] Zhang G, Shen $Y$, Wang L. Global anti-synchronization of a class of chaotic memristive neural networks with time-varying delays. Neural Netw 2013;46:1-8.

[26] Wu A, Zeng Z. Anti-synchronization control of a class of memristive recurrent neural networks. Commun Nonlinear Sci Numer Simul 2013;18:373-85.

[27] Wang W, Yu M, Luo X, Liu L, Yuan M, Zhao W. Synchronization of memristive BAM neural networks with leakage delay and additive time-varying delay components via sampled-data control. Chaos Solitons Fract 2017;104:84-97.

[28] Liu D, Zhu S, Sun K. Global anti-synchronization of complex-valued memristive neural networks with time delays. IEEE Trans Cybern 2018:99:1-13.

[29] Zheng M, Li X, Peng H, Wang W, Xiao J, Yang Y, Zhao H, Ren J. Finite-time synchronization of complex dynamical networks with multi-links via intermittent controls. Eur Phys J B 2016;89:1-12.

[30] Yu M, Wang W, Yuan M, Luo X, Liu L. Exponential antisynchronization control of stochastic memristive neural networks with mixed time-varying delays based on novel delay-dependent or delay-independent adaptive controller Math Probl Eng 2017;2017:1-16.

[31] Cai $H$, Huang L. Functional differential inclusions and dynamic behaviors for memristor-based BAM neural networks with time-varying delays. Commun Nonlinear Sci Numer Simul 2014:19:1279-300

[32] Syed M, Saravanakumar R, Cao J. New passivity criteria for memristor-based neutral-type stochastic BAM neural networks with mixed time-varying delays. Neurocomputing 2016;171:1533-47.

[33] Anbuvithya R, Mathiyalagan K, Sakthivel R, Prakash P. Non-fragile synchronization of memristive BAM networks with random feedback gain fluctuations. Commun Nonlinear Sci Numer Simul 2015;29:427-40.

[34] Mathiyalagan K, Park J, Sakthivel R. Synchronization for delayed memristive BAM neural networks using impulsive control with random nonlinearities. Appl Math Comput 2015:259:967-79.

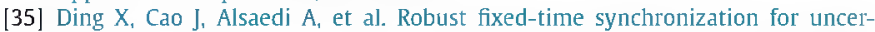
tain complex-valued neural networks with discontinuous activation functions. Neural Netw Off JInt Neural Netw Soci 2017:90:42.

[36] Yang X, Cao J, Yu W. Exponential synchronization of memristive Cohen-Grossberg neural networks with mixed delays. Cogn Neurodyn 2014;8:239-49.

[37] Gao J, Zhu P, Ahmed A, Fuad A, Tasawar H. A new switching control for finitetime synchronization of memristor-based recurrent neural networks. Neural Netw 2017:86:1-9.

[38] Shi $Y$, Zhu P. Finite-time synchronization of stochastic memristor-based delayed neural networks. Neural Comput Appl 2016:1-9.

[39] Zhao H, Li L, Peng H, Xiao J, Yang Y, Zheng M. Impulsive control for synchronization and parameters identification of uncertain multi-links complex network. Nonlinear Dyn 2016:83:1437-51.

[40] Xiao J, Zhong S, Li Y, Xu F. Finite-time Mittag-Leffler synchronization of fractional-order memristive BAM neural networks with time delays. Neurocomputing 2017;219:431-9.

[41] Pal D, Samanta GP, Mahapatra GS. Selective harvesting of two competing fish species in the presence of toxicity with time delay. Appl Math Comput 2017;313:74-93.

[42] Sun GQ, Wang SL, Ren Q, et al. Erratum: effects of time delay and space on herbivore dynamics: linking inducible defenses of plants to herbivore outbreak. Sci Rep 2015;5:11246.

[43] Li L, Jin Z, Li ]. Periodic solutions in a herbivore-plant system with time delay and spatial diffusion. Appl Math Modell 2016:40(7-8):4765-77.

[44] Upadhyay RK, Agrawal R. Dynamics and responses of a predator-prey system with competitive interference and time delay. Nonlinear Dyn 2015:83(1-2):1-17.

[45] Wen $S_{1}$ Yu X, Zeng Z. Event-triggering load frequency control for multiarea power systems with communication delays. IEEE Trans ]nd Electron 2015;63:1308-17

[46] Li N, Cao J. New synchronization criteria for memristor-based networks: adaptive control and feedback control schemes. Neural Netw 2015;61:1-9.

[47] Wen S, Zeng Z, Huang T. New criteria of passivity analysis for fuzzy time-delay systems with parameter uncertainties. IEEE Trans Fuzzy Syst 2015:23:2284-301

[48] Yang $X_{1}$ Cao J, Lu J. Synchronization of coupled neural networks with random coupling strengths and mixed probabilistic time-varying delays. Int J Robust Nonlinear Control 2013:23:2060-81

[49] Song Y. Wen S. Synchronization control of stochastic memristor-based neural networks with mixed delays. Neurocomputing 2015;156:121-8.

[50] Bao H, Park J, Cao J. Exponential synchronization of coupled stochastic memristor-based neural networks with time-varying probabilistic delay coupling and impulsive delay. IEEE Trans Neural Netw Learn Syst 2015;27:190-201.

[51] Li R, Cao ], Tu Z. Passivity analysis of memristive neural networks with probabilistic time-varying delays. Neurocomputing 2016;191:249-62.

[52] Song Q Zhao Z. Stability criterion of complex-valued neural networks with both leakage delay and time-varying delays on time scales. Neurocomputing 2016:171:179-84

[53] Li R, Cao J. Stability analysis of reaction-diffusion uncertain memristive neural networks with time-varying delays and leakage term. Appl Math Comput 2016:278:54-69.

[54] Wang F, Liu M. Global exponential stability of high-order bidirectional associative memory (BAM) neural networks with time delays in leakage terms. Neurocomputing 2016; 177:515-28.

[55] Wu A, Wen S, Zeng Z. Synchronization control of a class of memristor-based recurrent neural networks. Inf Sci 2012;183:106-16. 
[56] Wang G, Shen Y. Exponential synchronization of coupled memristive neural networks with time delays. Neural Comput Appl 2014;24:1421-30.

[57] Wu A, Zeng $Z$, Zhu $X$, Zhang J. Exponential synchronization of memristor-based recurrent neural networks with time delays. Neurocomputing 2011;74:3043-50.

[58] Bao H. Park J, Cao J. Exponential synchronization of coupled stochastic memristor-based neural networks with time-varying probabilistic delay coupling and impulsive delay. IEEE Trans Neural Netw Learn Syst 2016;27:190-201.
[59] Zhao H, Li L, Peng H, Kurths J, Xiao J, Yang Y. Anti-synchronization for stochastic memristor-based neural networks with non-modeled dynamics via adaptive control approach. Eur Phys J B 2015:88:1-10.

[60] Meng Z, Xiang Z. Stability analysis of stochastic memristor-based recurrent neural networks with mixed time-varying delays. Neural Comput Appl 2016:1-13.

Post-print standardized by MSL Academic Endeavors, the imprint of the Michael Schwartz Library at Cleveland State University, 2019 